\title{
Claves para la identificación de los peces de las aguas continentales e insulares de Costa Rica. Parte I: Familias
}

\author{
Arturo Angulo $^{1,2}$, Ana Rosa Ramirez-Coghi ${ }^{1}$ (D) Myrna López $^{1}$ (D) \\ 1. Museo de Zoología, Centro de Investigación en Biodiversidad y Ecología Tropical (CIBET), Universidad de Costa \\ Rica. 11501-2060, San Pedro de Montes de Oca, San José, Costa Rica; arturo.angs@gmail.com; \\ ana.ramirezcoghi@ucr.ac.cr; myrna.lopezbussing@gmail.com \\ 2. Centro de Investigación en Ciencias del Mar y Limnología (CIMAR), Universidad de Costa Rica. 11501-2060, San \\ Pedro de Montes de Oca, San José, Costa Rica.
}

Recibido 31-VIII-2020 • Corregido 18-IX-2020 • Aceptado 28-IX-2020 DOI: https://doi.org/10.22458/urj.v13i1.3145

\begin{abstract}
Identification keys for the fish of continental and insular waters of Costa Rica. Part I: Families." Introduction: Knowledge about the taxonomic composition of the Costa Rican freshwater fish fauna has changed substantially in the last two decades. Not only due to the addition of new species and supra-specific taxa, but also due to rearrangements and taxonomic changes at the different hierarchical levels. The available taxonomic keys were published more than 20 years ago and are outdated. Objective: To provide up-to-date information, in a friendly language, for the identification of all Costa Rican families of continental and insular fishes. Methods: We used specialized literature, museum collections, and recently collected specimens to update the available keys based on a 2013 family list. Results: The key covers 53 families. The illustrated dichotomous key is based on external characters that are relatively "easy" to identify. We also include information about species composition, general distribution and habitat use. Conclusion: This key allows the identification of $\mathbf{2 7 4}$ fish species to family level, as a basis for further identification.
\end{abstract}

Keywords: Diversity, ichthyofauna, freshwater fishes, Central America, Neotropical region.
RESUMEN. Introducción: El conocimiento sobre la composición taxonómica de la fauna de peces de agua dulce de Costa Rica ha cambiado sustancialmente en las últimas dos décadas. No solamente por la adición de nuevas especies y táxones supra-específicos, sino también por reordenamientos y cambios taxonómicos en los diferentes niveles jerárquicos. Las claves taxonómicas disponibles se publicaron hace más de 20 años y están desactualizadas. Objetivo: Brindar información actualizada, en lenguaje sencillo, para la identificación de todas las familias costarricenses de peces continentales e insulares. Métodos: Utilizamos literatura especializada, colecciones de museos y especímenes recolectados recientemente para actualizar las claves disponibles tomando como referencia una lista de familias del 2013. Resultados: La clave dicotómica ilustrada, que cubre 53 familias, se basa en características externas relativamente fáciles de identificar. También incluimos información sobre la composición de especies, distribución general y uso del hábitat. Conclusión: Esta clave permite la identificación de 274 especies de peces costarricenses a nivel de familia, como base para su identificación a categorías más bajas.

Palabras clave: Diversidad, ictiofauna, peces de aguas dulce, Centroamérica, región neotropical.

Desde la publicación del libro de Bussing (1998): "Peces de las aguas continentales de Costa Rica", una de las obras más importantes estableciendo la base para la investigación ictiológica en aguas dulces a nivel nacional y regional, mucho se ha avanzado en lo referente al conocimiento general (sistemática, taxonomía, ecología, biogeografía, etc.) sobre este componente de la biodiversidad costarricense. Posteriormente, Angulo et al. (2013) publicaron el listado de la fauna íctica de las aguas continentales e insulares de Costa Rica más actualizado a la fecha, en el cual se incluyeron 250 especies. Con esta contribución se adicionaron más de 100 especies al elenco originalmente reportado, descrito y discutido por Bussing (1998), lo que representó un incremento de alrededor de un $75 \%$ en el número total de especies listadas para el país (i.e., 134). 
En los últimos 7-8 años, diversas investigaciones puntuales han revelado además complejos patrones de diversidad críptica en diferentes grupos de peces con distribución en Costa Rica, así como áreas del país y grupos taxonómicos con conocimiento insuficiente, principalmente en cuanto a su diversidad general e intrínseca, patrones de distribución, relaciones evolutivas, entre otros. Tales avances se han visto reflejados en diversas publicaciones científicas incrementando el número total de especies reconocidas para el país (e.g., Schmitter-Soto, 2017; Angulo et al., 2015; 2020 en prep., \& Álvarez-Calderón et al., 2018; entre otros), ampliando los ámbitos de distribución vertical y/o geográfico conocido para varias especies (e.g. Angulo et al., 2015; 2017; 2018a, b; Arguedas, 2019; Rodríguez-Arias et al., 2019, \& Angulo et al., 2020) e incluso añadiendo/describiendo nuevas especies para la ciencia, algunas de ellas con localidades tipo en Costa Rica (e.g., Matamoros et al., 2013; Angulo \& Gracian-Negrete, 2013; Angulo et al., 2018a, \& Ota et al., 2020), entre otros. Dado esto, el conocimiento sobre la composición taxonómica de la fauna íctica (de aguas continentales e insulares) costarricense ha cambiado, de forma sustancial, en los últimos años; no solo por la adición de múltiples especies y taxones supra-específicos sino también como consecuencia de diversos rearreglos y cambios taxonómicos en los diferentes niveles jerárquicos, principalmente géneros, pero también familias y órdenes (Angulo et al., 2013; 2015; Nelson et al., 2016; Angulo et al., 2017; 2018; 2020 en prep.), esto tomando como base la publicación de Bussing (1998).

Bussing (1998) listó inicialmente un total de 34 familias de peces habitando las aguas continentales e insulares de Costa Rica, no obstante, Angulo et al. (2013; 2015; 2017; 2018a; 2020, en prep.) reportan un total de 53 familias. La clave provista por Bussing (1998), dirigida inicialmente a facilitar la identificación de estos grupos taxonómicos supra-específicos (i.e., familias), pero incluyendo también claves genéricas y específicas (siendo la única herramienta de su tipo disponible a la fecha) se encuentra por tanto desactualizada; esto ya que no incluye la totalidad de familias, géneros y especies actualmente listada(o)s y/o reconocida(o)s para el país. Lo anterior imposibilita o dificulta, en la actualidad, una adecuada identificación a tales niveles taxonómicos, principalmente al considerar la gran cantidad de taxones que estarían siendo omitidos, entre otros problemas (e.g., cambios o modificaciones a nivel nomenclatural).

En respuesta a lo anterior, el objetivo de la presente contribución, parte de una serie de trabajos interrelacionados y complementarios sobre el tema, es proveer, a aquellas personas interesadas (i.e., estudiantes, académicos, investigadores, aficionados y otros), información actualizada y en un lenguaje amigable, que permita la identificación de la totalidad de especies y grupos de especies de peces (a nivel de familia) habitando las aguas continentales (e insulares) del país. En esta primera contribución se ofrece una clave enfocada en los grupos taxonómicos supraespecíficos (i.e., familias), esperando en contribuciones subsecuentes entrar en detalle en aquellos grupos con mayor diversidad y representatividad en aguas dulces costarricenses. Para aquellas familias mono- o bi-específicas (i.e., representadas en Costa Rica por una o dos especies, respectivamente) se incluyen en este tratado los nombres de las especies correspondientes (así como información distribucional y/o de identificación), eliminando así la necesidad de tratados particulares o dirigidos a tales grupos. Se incluye además información sobre nombres comunes y distribución. Por último, se presenta una guía ilustrada (Figs. 1-4) para facilitar la identificación de los grupos tratados, complementado así la información textual brindada en la clave. 


\section{MATERIALES Y MÉTODOS}

El elenco o composición de grupos taxonómicos (i.e., familias) a considerar en esta contribución se basa principalmente en el listado provisto por Angulo et al. (2013) con adiciones y modificaciones según Angulo et al. (2015; 2017; 2018, \& 2020), Álvarez-Calderón et al. (2018) y Fricke et al. (2020). Un listado actualizado para el país, reflejando tales cambios se encuentra en preparación por el mismo autor (y colaboradores) y será formalmente publicado en breve. El nombre válido de cada familia, su composición específica y clasificación superior (a nivel de orden), sigue a Fricke et al. (2020). Los nombres comunes y distribución (geográfica) de los diferentes taxones siguen a Angulo (2013), Angulo et al. (2013; 2015; 2017; 2018a, b; 2020), Robertson \& Allen (2015) y Robertson \& Van Tassell (2019). Las ilustraciones provistas (Figs. 1-9), complementando la clave escrita, constituyen en su mayoría modificaciones y/o adaptaciones de aquellas previamente publicadas por Bussing (1998) y Nelson et al. (2016), con algunas adiciones inéditas.

La clave presenta opciones dicotómicas (i.e., duales: a o b, por cada paso, mutuamente excluyentes) y está basada principalmente en caracteres anatómicos externos de relativamente fácil identificación y observación. En las figuras 5-8 se ilustran y definen, a modo de guía o referencia, algunos de estos caracteres, condiciones y/o estructuras, así como las principales variantes y/o condiciones alternativas observadas, esto como complemento a lo detallado en el texto de la clave. De igual forma, y a efectos de facilitar su uso, se hace la referencia a la estructura o carácter en cuestión, ilustrado en la figura, en el(los) paso(s) correspondiente(s) o aplicable(s) de la clave. Los datos utilizados para generar la clave provienen de dos fuentes principales: (1) literatura científica, a lo que vale resaltar el aporte de referencias base, incluyendo los trabajos de Bussing (1998), Robertson \& Allen (2015) y Robertson \& Van Tassell (2019), y (2) revisión, caracterización y análisis de material museográfico de referencia parte del acervo de la colección ictiológica del museo de Zoología de la Universidad de Costa Rica (UCR), ver Angulo et al. (2016). En ese sentido, el material de la colección fue revisado y caracterizado siguiendo los protocolos y terminología provistos por Bussing (1998) y a como detallado en publicaciones específicas para determinados grupos taxonómicos (e.g., Angulo \& Gracian-Negrete 2013; Angulo et al., 2018a, b); lo cual incluye conteos, mediciones y otras observaciones realizadas sobre el lado izquierdo de los ejemplares. Datos y observaciones, en la clave, referentes al patrón de coloración de los diferentes taxones en vida fueron tomados y realizadas en campo y comparadas con los datos publicados por Bussing (1998), Robertson \& Allen (2015) y Robertson \& Van Tassell (2019).

\section{RESULTADOS}

En esta revisión se incluyeron un total de 53 familias en 26 órdenes agrupando 274 especies (Cuadro 1). El número total de especies reportado incluye registros publicados en revisiones previas (e.g., Angulo et al., 2015; 2017; 2018; 2020, \& Álvarez-Calderón et al., 2018) así como datos sin publicar. Del total de familias listadas 11 son, según la propuesta de Myers (1949), dulceacuícolas primarias (i.e., dulceacuícolas estrictas), 7 son dulceacuícolas secundarias (i.e., usualmente restrictas a ambientes dulceacuícolas, pero con tolerancia a condiciones variables de salinidad) y 35 son periféricas (i.e., típicamente marinas o asociadas a ambientes costero-estuarinos con capacidad de incursionar en ambientes dulceacuícolas) (Cuadro 1). La clave resultante se presenta a continuación: 


\section{CUADRO 1}

Familias incluidas en la clave

\begin{tabular}{|c|c|c|c|c|}
\hline Orden & Familia & \# Especies & Tolerancia & \# Figura \\
\hline Albuliformes & Albulidae & $1^{*}$ & Per & $1 \mathrm{~A}$ \\
\hline \multirow[t]{2}{*}{ Anguilliformes } & Anguillidae & $1^{*}$ & Per & $1 \mathrm{~B}$ \\
\hline & Ophichthidae & $2^{*}$ & Per & $1 C$ \\
\hline Atheriniformes & Atherinopsidae & $7^{*}$ & Per & $1 \mathrm{D}$ \\
\hline \multirow[t]{2}{*}{ Beloniformes } & Belonidae & $2^{*}$ & Per & $1 \mathrm{E}$ \\
\hline & Hemiramphidae & $2 *$ & Per & $1 \mathrm{~F}$ \\
\hline \multirow[t]{2}{*}{ Blenniiformes } & Blenniidae & $1^{*}$ & Per & $1 G$ \\
\hline & Dactyloscopidae & $1^{*}$ & Per & $1 \mathrm{H}$ \\
\hline Carcharhiniformes & Carcharhinidae & $1 *$ & Per & 11 \\
\hline \multirow[t]{2}{*}{ Centrarchiformes } & Centrarchidae & 1 & $\mathrm{Sec}$ & $1 \mathrm{~J}$ \\
\hline & Kyphosidae & 1 & Per & $1 K$ \\
\hline \multirow[t]{5}{*}{ Characiformes } & Bryconidae & 2 & Pri & $1 \mathrm{~L}$ \\
\hline & Characidae & 18 & Pri & $1 \mathrm{M}$ \\
\hline & Curimatidae & 1 & Pri & $1 \mathrm{~N}$ \\
\hline & Erythrinidae & 1 & Pri & $2 \mathrm{~A}$ \\
\hline & Lebiasinidae & 1 & Pri & $2 \mathrm{~B}$ \\
\hline Cichliformes & Cichlidae & 27 & $\mathrm{Sec}$ & $2 \mathrm{C}$ \\
\hline \multirow[t]{2}{*}{ Clupeiformes } & Clupeidae & $4^{*}$ & Per & $2 \mathrm{D}$ \\
\hline & Engraulidae & $5^{*}$ & Per & $2 \mathrm{E}$ \\
\hline Cypriniformes & Cyprinidae & 2 & Pri & $2 \mathrm{~F}$ \\
\hline \multirow[t]{3}{*}{ Cyprinodontiformes } & Anablepidae & 1 & $\mathrm{Sec}$ & $2 \mathrm{G}$ \\
\hline & Poeciliidae & 26 & $\mathrm{Sec}$ & $2 \mathrm{H}$ \\
\hline & Rivulidae & 7 & $\mathrm{Sec}$ & 21 \\
\hline \multirow[t]{2}{*}{ Elopiformes } & Elopidae & $1^{*}$ & Per & $2 \mathrm{~J}$ \\
\hline & Megalopidae & $1^{*}$ & Per & $2 \mathrm{~K}$ \\
\hline Gobiesociformes & Gobiesocidae & $3^{*}$ & Per & $2 \mathrm{~L}$ \\
\hline \multirow[t]{3}{*}{ Gobiiformes } & Eleotridae & $16^{*}$ & Per & $2 \mathrm{M}$ \\
\hline & Gobiidae & $24 *$ & Per & $2 \mathrm{~N}$ \\
\hline & Microdesmidae & $1^{*}$ & Per & 20 \\
\hline \multirow[t]{2}{*}{ Gymnotiformes } & Gymnotidae & 2 & Pri & $2 \mathrm{P}$ \\
\hline & Hypopomidae & 1 & Pri & $2 Q$ \\
\hline Lepisosteiformes & Lepisosteidae & 1 & $\mathrm{Sec}$ & $3 A$ \\
\hline Mugiliformes & Mugilidae & $6^{*}$ & Per & $3 B$ \\
\hline \multirow[t]{9}{*}{ Perciformes } & Carangidae & $10^{*}$ & Per & $3 C$ \\
\hline & Centropomidae & $11^{*}$ & Per & $3 D$ \\
\hline & Gerreidae & $14^{*}$ & Per & $3 \mathrm{E}$ \\
\hline & Haemulidae & $8^{*}$ & Per & $3 F$ \\
\hline & Labridae & $3 *$ & Per & $3 G$ \\
\hline & Lutjanidae & $8^{*}$ & Per & $3 \mathrm{H}$ \\
\hline & Polynemidae & $2 *$ & Per & 31 \\
\hline & Sciaenidae & $5^{*}$ & Per & $3 \mathrm{~J}$ \\
\hline & Serranidae & $1 *$ & Per & $3 K$ \\
\hline
\end{tabular}




\begin{tabular}{lllll} 
Pleuronectiformes & Achiridae & $8^{*}$ & Per & $3 \mathrm{~L}$ \\
& Paralichthyidae & $4^{*}$ & Per & $4 \mathrm{~A}$ \\
Pristiformes & Pristidae & $2^{*}$ & Per & $4 \mathrm{~B}$ \\
Salmoniformes & Salmonidae & $1^{*}$ & Per & $4 \mathrm{C}$ \\
Siluriformes & Ariidae & $7^{*}$ & Per & $4 \mathrm{D}$ \\
& Heptapteridae & 5 & Pri & $4 \mathrm{E}$ \\
& Loricaridae & 3 & Pri & $4 \mathrm{~F}$ \\
Synbranchiformes & Trichomycteridae & 1 & Pri & $4 \mathrm{G}$ \\
Syngnathiformes & Synbranchidae & 2 & Sec & $4 \mathrm{H}$ \\
Tetraodontiformes & Syngathidae & $5^{*}$ & Per & $4 \mathrm{I}$ \\
\hline
\end{tabular}

*El número de especies listado no necesariamente corresponde al número total de especies reportadas para el país e incluye solo aquellas especies con registros confirmados en ambientes dulceacuícolas (i.e., excluye las especies típicamente marinas).

**Para cada familia se incluye el número total de especies (\#Especies), tolerancia a la salinidad (Tolerancia: Clasificadas como Pri=Primarias, Sec=Secundarias y Per=Periféricas) y referencia a la figura (\#Figura) correspondiente ilustrando el patrón general corporal.

\section{Clave para la identificación de las familias de peces de las aguas continentales e insulares de Costa Rica}

1a. Cuerpo anguiliforme (i.e., con forma de anguila o serpiente; Figs. $1 \mathrm{~B}-\mathrm{C}, 2 \mathrm{P}-\mathrm{Q}, 4 \mathrm{H}$ ), bastante alargado (la longitud total del cuerpo es mucho mayor que su altura máxima; ver Figs. 5A, 5C); escamas ausentes o muy reducidas; aletas pélvicas ausentes; aletas dorsal, anal y/o caudal ausentes, si presentes generalmente reducidas o fusionadas [excepto la anal en Gymnotidae (Fig. 2P) e Hypopomidae (Fig. 2Q) la cual es bastante conspicua]... 2

1b. Cuerpo de forma variable, muy alargado [e.g., Microdesmidae (Fig. 2O) y Syngnathidae (Fig. 4I)], ligeramente alargado (e.g., Figs. 1D-F), u oval o redondeado (la longitud total del cuerpo no es mucho mayor que su altura máxima; ver Figs. 5A, 5C), nunca anguliforme (i.e., no con forma de anguila o serpiente); escamas generalmente presentes, ausentes en algunos casos [e.g., Ariidae (Fig. 4D), Heptapteridae (Fig. 4E) y Trichomycteridae (Fig. 4G)] o modificadas como placas o anillos en otros [e.g., en Loricariidae (Figs. 4F, 6F) y Syngnathidae (Figs. 4I, 6E)]; aletas pélvicas generalmente presentes [ausentes en Syngnathidae (Fig. 4I) y Tetraodontidae (Fig. 4J)]; aletas dorsal, anal y caudal presentes [ver Figs. 5 y 7 ; aleta anal ausente en Syngnathidae (Fig. 4I)] ... 6

2a. Aletas pectorales, dorsal, anal y caudal ausentes o muy reducidas, visibles solo como un pliegue en la piel ... Synbranchidae (representada en las aguas continentales e insulares de Costa Rica por dos especies, conocidas como "Anguilas de pantano, Anguilas de suampo, Anguilas de lodo, Anévilas, Anguilas falsas o Culebras" - Synbranchus marmoratus y Ophisternon aenigmaticum, distribuidas en todo el país; Fig. $4 \mathrm{H}$ )

2b. Aletas pectorales, dorsal y anal presentes [dorsal ausente en Gymnotidae (Fig. 2P) e Hypopomidae (Fig. 2Q)], la caudal ausente o fusionada con las aletas dorsal y anal ... $\mathbf{3}$

3a. Cuerpo robusto, cilíndrico; aleta dorsal presente; aleta anal moderadamente larga, la longitud de su base corresponde a un $40-60 \%$ de la longitud total del cuerpo ... 4

3b. Cuerpo comprimido lateralmente, con forma de cuchillo (Figs. 2P-Q); aleta dorsal ausente; aleta anal bastante larga, la longitud de su base corresponde a un $60-80 \%$ de la longitud total del cuerpo ... 5 
4a. Mandíbula inferior proyectada anteriormente (sobrepasa la mandíbula superior en el plano vertical; Fig. 8J); labios bien desarrollados, carnosos; comisura bucal (o límite posterior de la boca) situado por debajo de la línea media del ojo, nunca posterior al (o detrás del) borde posterior del ojo; ojo bien desarrollado, relativamente grande, su diámetro equivale a un 30-50\% o más de la longitud de la boca; aleta dorsal moderadamente larga, 50-65\% de la longitud total del cuerpo ... Anguillidae (representada en las aguas continentales e insulares de Costa Rica por una única especie, la "Anguila, Anguila de mar, Anguila verdadera o Anguila Americana" - Anguilla rostrata, distribuida en la vertiente Atlántica; Fig. 1B)

4b. Mandíbula superior proyectada anteriormente (sobrepasa la mandíbula inferior en el plano vertical; Fig. 8I); labios poco desarrollados, no carnosos; comisura bucal (o límite posterior de la boca) situado posterior al (o detrás del) borde posterior del ojo; ojo de tamaño mediano, su diámetro equivale a menos de un $30 \%$ de la longitud de la boca; aleta dorsal extremadamente larga, 60-80\% de la longitud total del cuerpo ... Ophichthidae (representada en las aguas continentales e insulares de Costa Rica por dos especies, conocidas como "Anguilas lombriz, Tiesos lombriz o Tiesos gusano" - Myrophis punctatus y M. vafer, presentes en ambientes costeros en el Atlántico y Pacífico, respectivamente; Fig. 1C)

5a. Mandíbula inferior proyectada anteriormente (sobrepasa la mandíbula superior en el plano vertical; Fig. 8J); dientes presentes, pequeños; aleta anal terminando en el extremo posterior del cuerpo ... Gymnotidae (representada en las aguas continentales e insulares de Costa Rica por dos especies, conocidas como "Peces cuchillo, Navajas, Machetes, Anguilas, Anguilas falsas o Madres de barbudo" - Gymnotus cylindricus y G. Maculosus, distribuidas en todo el país; Fig. 2P)

5b. Mandíbula superior proyectada anteriormente (sobrepasa la mandíbula inferior en el plano vertical; Fig. 8I); dientes ausentes (sin dientes); aleta anal terminando mucho antes del extremo posterior del cuerpo, siendo evidente una especie de cola o proyección caudal ... Hypopomidae (representada en las aguas continentales e insulares de Costa Rica por una única especie, el "Pez cuchillo, Navaja, Machete, Anguila, Anguila falsa, Madre de barbudo o Cuchillo aplanado" Brachyhypopomus occidentalis, distribuida en la región Caribe Sur; Fig. 2Q)

6a. Escamas presentes, del tipo placoideas (Fig. 6A); cinco aberturas branquiales (Fig. 1I); aletas pélvicas en machos modificadas como órganos copulatorios (pterigopodios; Fig. 9E); aleta caudal heterocerca (Fig. 7A) ... 7

6b. Escamas presentes o ausentes, si presentes, del tipo ganoideas (Fig. 6B), cicloideas (Fig. 6C) o ctenoideas (Fig. 6D), nunca placoideas, en algunos casos modificadas como placas o anillos [e.g., en Loricariidae (Figs. 4F, 6F) y Syngnathidae (Figs. 4I, 6E)]; una única abertura branquial situada en el extremo posterior de la cabeza; aletas pélvicas en machos no modificadas como órganos copulatorios; aleta caudal heterocerca abreviada (en Lepisosteidae; Fig. 7B) u homocerca (en el resto de familias), variando en su estructura y forma (Figs. 7C-F) ... 8

7a. Cuerpo robusto, no comprimido dorso-ventralmente; hocico relativamente corto, menor que la altura máxima del cuerpo, no en forma de doble sierra ... Carcharhinidae (representada en las aguas continentales e insulares de Costa Rica por una única especie, el "Tiburón toro, Madrecazón o Tiburón sarda" - Carcharhinus leucas, presente en ambientes costeros en el Atlántico y Pacífico; Fig. 1I)

7b. Cuerpo deprimido (comprimido dorso-ventralmente); hocico alargado, su longitud mayor (más de dos veces) que la altura máxima del cuerpo, en forma de doble sierra y con numerosos dientes laterales ... Pristidae (representada en las aguas continentales e insulares de Costa Rica por dos 
especies, conocidas como "Peces sierra, Pejesierras, Peces peine o Pejepeines" - Pristis pectinata y P. pristis, presentes en ambientes costeros en el Atlántico y Pacífico; Fig. 4B)

8a. Cuerpo asimétrico, i.e., pigmentación y ambos ojos presentes en un solo lado del cuerpo (Figs. $3 \mathrm{~L}, 4 \mathrm{~A})$; cuerpo fuertemente comprimido lateralmente ... 9

8b. Cuerpo simétrico, i.e., pigmentación y ojo presentes en ambos lados del cuerpo; cuerpo no fuertemente comprimido lateralmente ... 10

9a. Cuerpo redondeado o elíptico (verticalmente alargado) en vista lateral; ojos y pigmentación presentes solo en el lado derecho del cuerpo; ojos y boca relativamente pequeños o reducidos; boca ondulada ... Achiridae (representada en las aguas continentales e insulares de Costa Rica por ocho especies, conocidas como "Lenguados, Lenguados redondos, Suelas, Suelas americanas, Lenguados derechos o Peces hoja”, presentes en ambientes costeros en el Atlántico y Pacífico; Fig. 3L)

9b. Cuerpo más o menos romboidal a ovalado (longitudinal u horizontalmente alargado) en vista lateral; ojos y pigmentación presentes solo en el lado izquierdo del cuerpo; ojos y boca relativamente grandes, no reducidos; boca curva ... Paralichthyidae (representada en las aguas continentales e insulares de Costa Rica por cuatro especies, conocidas como "Lenguados, Peces planos o Peces Hoja", presentes en ambientes costeros en el Atlántico y Pacífico; Fig. 4A)

10a. Cuerpo cubierto por una serie de placas (no escamas) o anillos óseos, formando una especie de "coraza" rígida (Figs. 6E-F) ... 11

10b. Cuerpo desnudo (sin escamas) o cubierto por escamas de tamaño y forma variable [ganoideas (Fig. 6B), cicloideas (Fig. 6C) o ctenoideas (Fig. 6D)], no por placas o anillos óseos ... 12

11a. Cuerpo delgado y bastante alargado, cubierto por una especie de "coraza" o "armadura" rígida compuesta por una serie de segmentos óseos a manera de anillos (Fig. 6E); boca terminal, pequeña, sin dientes ni barbillas, al extremo de un hocico largo y tubular (Fig. 6E); ojos en posición lateral; aletas pélvicas ausentes ... Syngnathidae (representada en las aguas continentales e insulares de Costa Rica por cinco especies, conocidas como "Peces pipa, Palos, Palitos, Trompeteros, Agujas o Lápices", presentes en ambientes costeros en el Atlántico y Pacífico; Fig. 4I)

11b. Cuerpo robusto a ligeramente alargado, cubierto por una especie de "coraza" o "armadura" rígida compuesta por una serie de segmentos óseos a manera de placas (Fig. 6F); boca ventral, en posición intermedia en el hocico (no terminal), con dientes, labios prominentes y barbillas, hocico no tubular; ojos en posición dorso-lateral; aletas pélvicas presentes ... Loricariidae (representada en las aguas continentales e insulares de Costa Rica por tres especies, conocidas como "Peces diablo, Arrisuacas, Plecos o Peces gato acorazados", distribuidas en todo el país, pero originalmente nativas de la región Pacífico Sur; Fig. 4F)

12a. Aleta dorsal adiposa presente (Figs. 1L-N, 2B, 4D-F, 5A) ... 13

12b. Aleta dorsal adiposa ausente (e.g., Figs. 1A, 2A, 5D) ... 19

13a. Cuerpo desnudo (sin escamas); barbillas o barbillones presentes (Figs. 4D-F) ... 14

13b. Cuerpo cubierto por escamas; barbillas o barbillones ausentes ... 15 

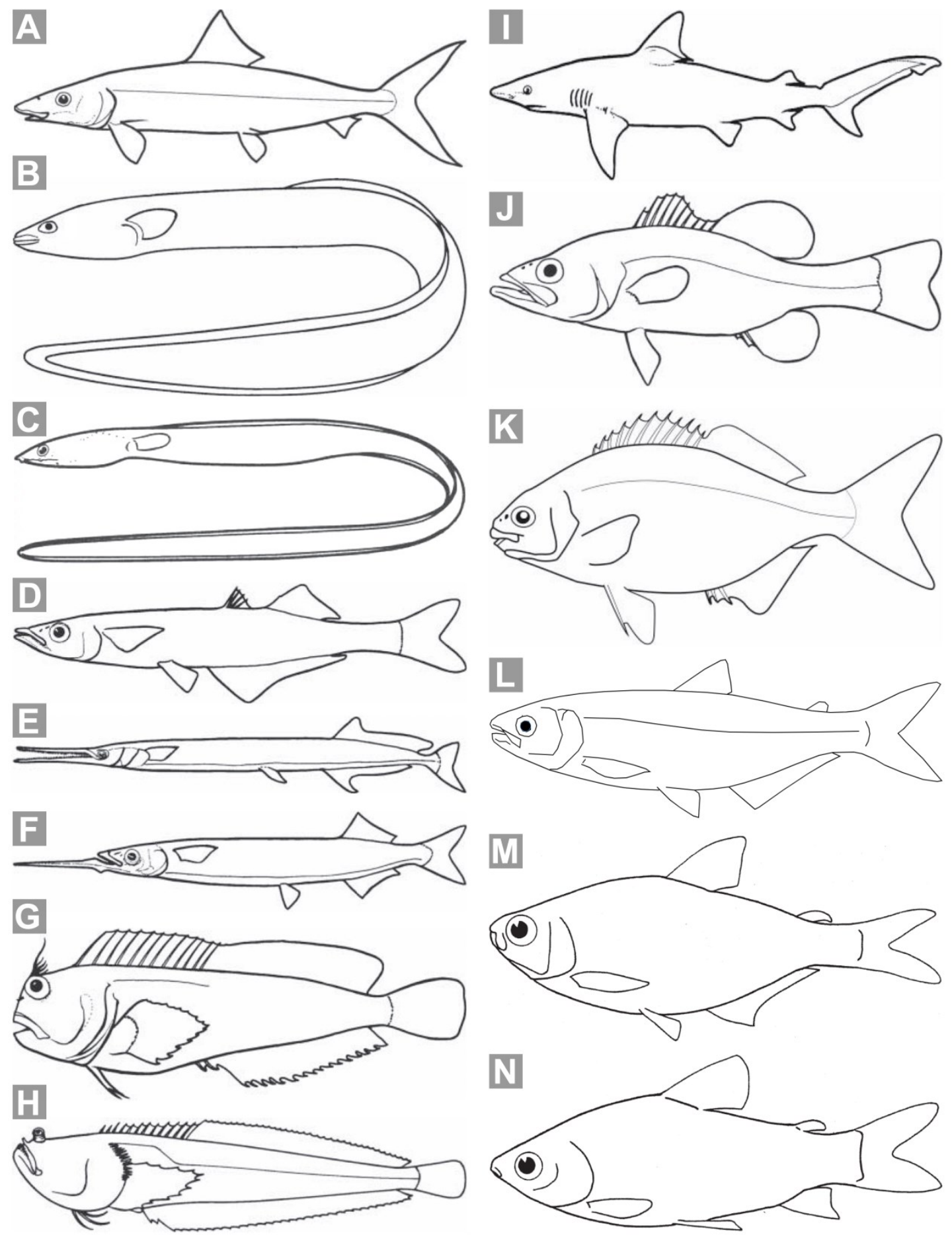

Fig. 1. Morfología externa generalizada/ilustrativa de un miembro de las familias Albulidae (A), Anguillidae (B), Ophichthidae (C), Atherinopsidae (D), Belonidae (E), Hemiramphidae (F), Blenniidae (G), Dactyloscopidae (H), Carcharhinidae (I), Centrarchidae (J), Kyphosidae (K), Bryconidae (L), Characidae (M) y Curimatidae (N). Modificado de Nelson et al. (2016); L: Ilustración inédita. 


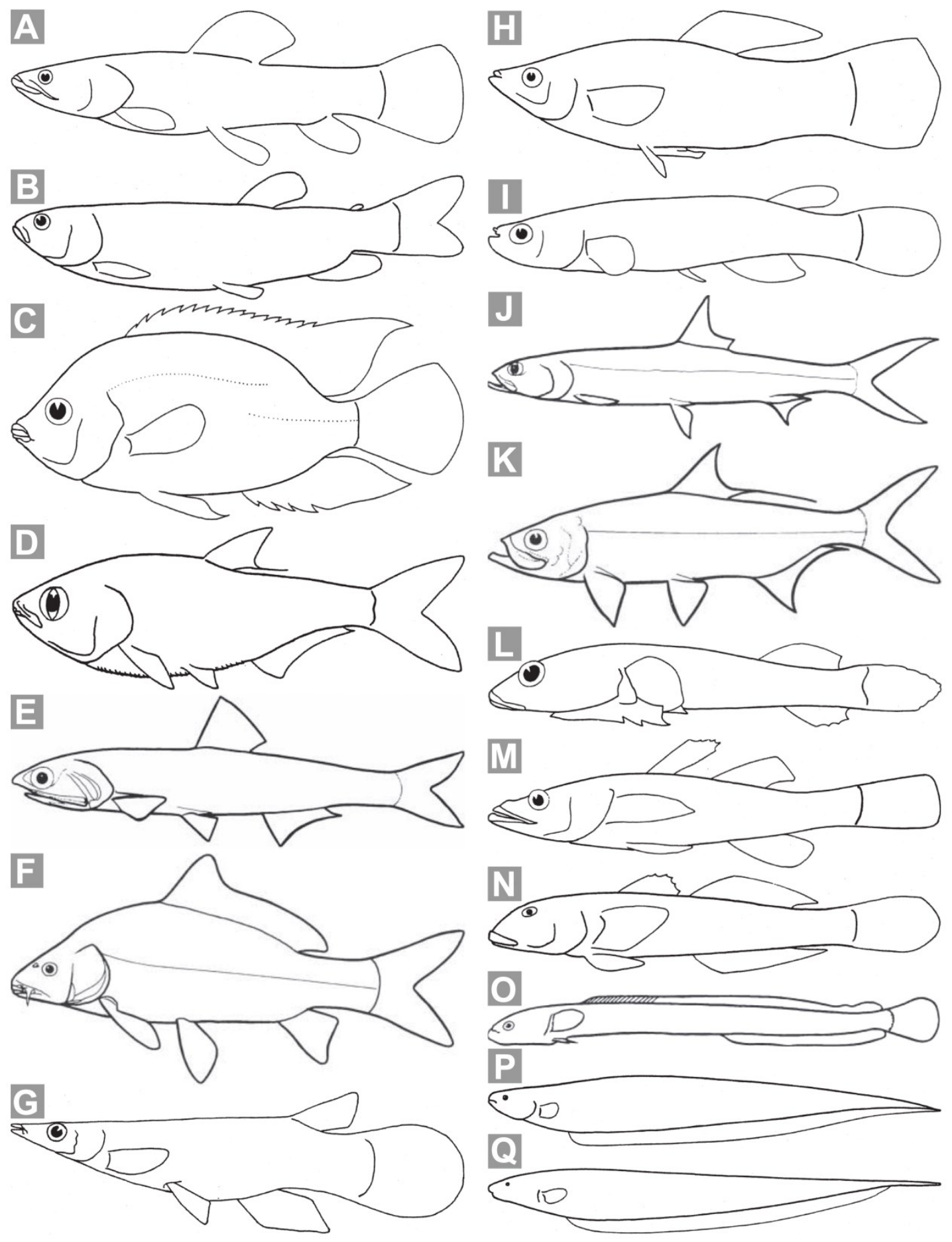

Fig. 2. Morfología externa generalizada/ilustrativa de un miembro de las familias Erythrinidae (A), Lebiasinidae (B), Cichlidae (C), Clupeidae (D), Engraulidae (E), Cyprinidae (F), Anablepidae (G), Poeciliidae (H), Rivulidae (I), Elopidae (J), Megalopidae (K), Gobiesocidae (L), Eleotridae (M), Gobiidae (N), Microdesmidae( O), Gymnotidae (P) e Hypopomidae (Q). Modificado de Nelson et al. (2016). 

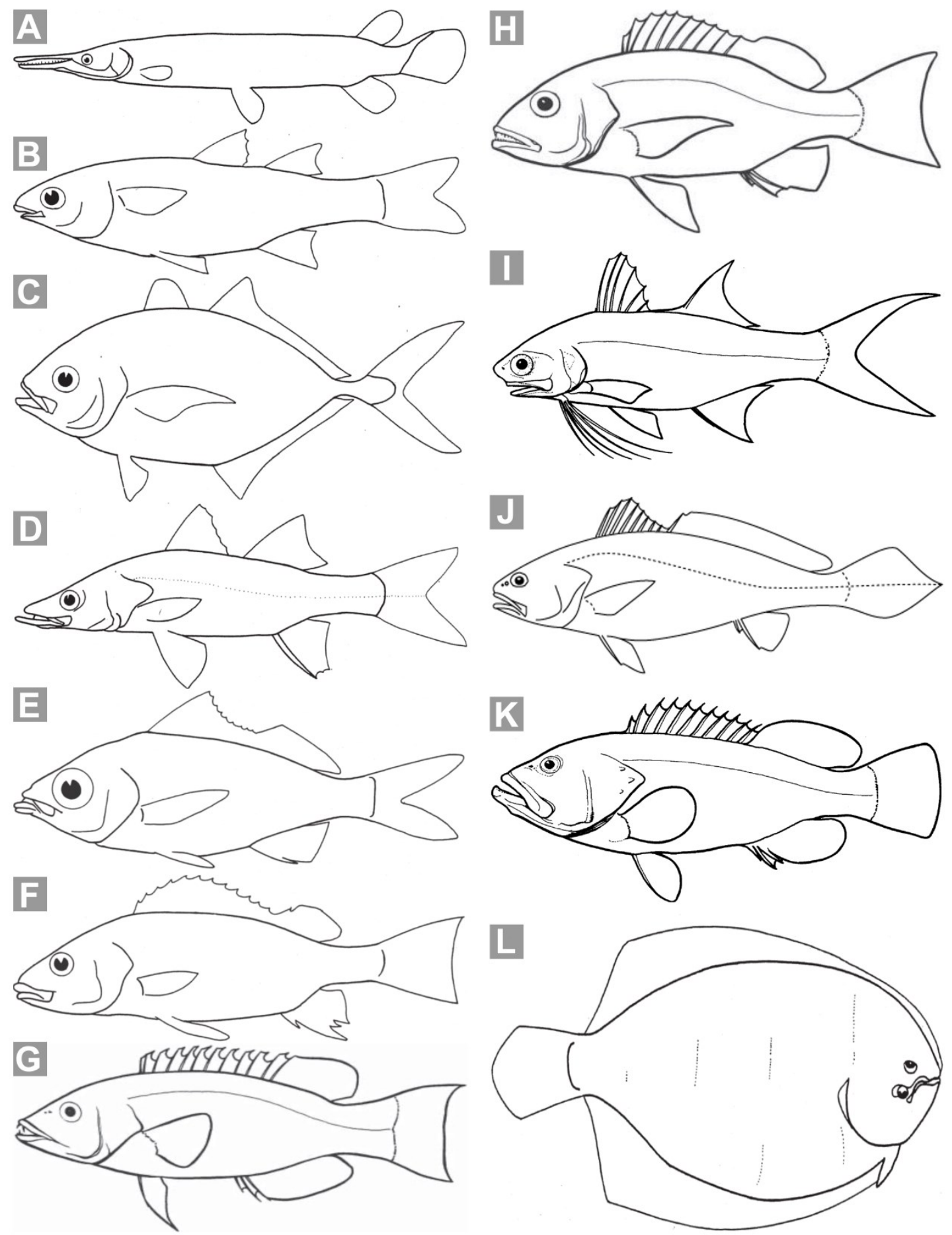

Fig. 3. Morfología externa generalizada/ilustrativa de un miembro de las familias Lepisosteidae (A), Mugilidae (B), Carangidae (C), Centropomidae (D), Gerreidae (E), Haemulidae (F), Labridae (G), Lutjanidae (H), Polynemidae (I), Sciaenidae (J), Serranidae (K) y Achiridae. Modificado de Nelson et al. (2016). 

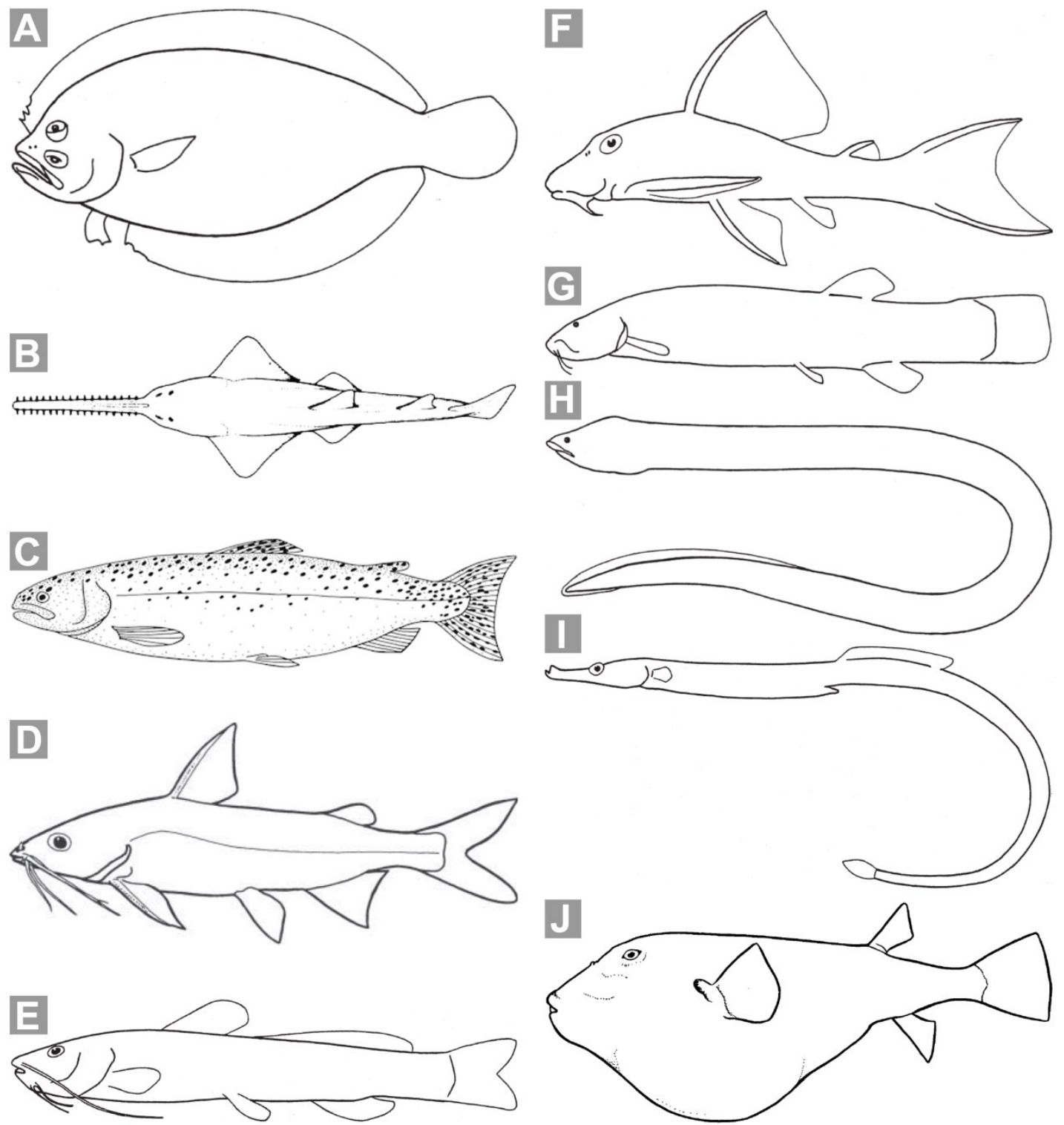

Fig. 4. Morfología externa generalizada/ilustrativa de un miembro de las familias Paralichthyidae (A), Pristidae (B), Salmonidae (C), Ariidae (D), Heptapteridae (E), Loricaridae (F), Trichomycteridae (G), Synbranchidae (H), Syngnathidae (I) y Tetraodontidae (J). Modificado de Nelson et al. (2016); C: Ilustración inédita.

14a. Aleta dorsal adiposa corta, su longitud mucho menor que la longitud de la cabeza; aleta anal con 16 o más radios (ver Figs. 5D y 9A) ... Ariidae (representada en las aguas continentales e insulares de Costa Rica por siete especies, conocidas como "Bagres, Cuminates, Congos o Peces gato", presentes en ambientes costeros en el Atlántico y Pacífico; Fig. 4D)

14b. Aleta dorsal adiposa larga, su longitud igual o mayor que la longitud de la cabeza; aleta anal con 15 o menos radios (ver Figs. 5D y 9A) ... Heptapteridae (representada en las aguas continentales e insulares de Costa Rica por cinco especies, conocidas como "Barbudos, Bagres, Chulines o Peces gato", distribuidas en todo el país; Fig. 4E)

15a. Aleta anal con 16 o más radios (ver Figs. 5D y 9A) ... 16

15b. Aleta anal con 15 o menos radios (ver Figs. 5D y 9A) ... 17 
16a. Mandíbula superior generalmente no proyectada anteriormente (no sobrepasa claramente la mandíbula inferior en el plano vertical); mandíbula superior con 1 o 2 filas de dientes multicúspides (Figs. 8D-G), en las especies de Roeboides es posible observar además dos pares de dientes externos en el premaxilar; adultos no sobrepasando los $15 \mathrm{~cm}$ de longitud total ... Characidae (representada en las aguas continentales e insulares de Costa Rica por 18 especies, conocidas como "Sardinas, Sardinas de río, Plateadas o Sabaletes", distribuidas en todo el país; Fig. 1M)

16b. Mandíbula superior proyectada anteriormente (sobrepasa claramente la mandíbula inferior en el plano vertical; Fig. 81); mandíbula superior con 3 o 4 filas de dientes multicúspides (Figs. 8D-H); adultos generalmente sobrepasando los $15 \mathrm{~cm}$ de longitud total ... Bryconidae (representada en las aguas continentales e insulares de Costa Rica por dos especies, conocidas como "Machacas, Sábalos o Sabaletes" - Brycon behreae y B. costaricensis, distribuidas en las vertiente Pacífica y Atlántica, respectivamente; Fig. $1 \mathrm{~L}$ )

17a. Cuerpo con varias pintas o manchas negras u oscuras (principalmente en la región dorsal) sobre un fondo verdoso o cobrizo, con una franja lateral roja o rosada; boca grande, con su extremo posterior alcanzando o sobrepasando el límite posterior del ojo en el plano vertical; escamas pequeñas, reducidas, más de 100 en la serie longitudinal; aleta caudal recortada o ligeramente bifurcada (Figs. 7E-F) ... Salmonidae (representada en las aguas continentales e insulares de Costa Rica por una única especie, introducida, la "Trucha o Trucha arcoiris" - Oncorhynchus mykiss, distribuida en zonas altas en ambas vertientes; Fig. 4C)

17b. Cuerpo plateado uniforme a ligeramente dorado, generalmente sin manchas oscuras o con una serie de pintas o manchas rojizas, amarillentas o marrones en los costados; boca pequeña, generalmente no alcanzando el límite anterior del ojo en el plano vertical; escamas relativamente grandes, conspicuas, menos de 40 en la serie longitudinal; aleta caudal completamente bifurcada (Fig. 7F) ... 18

18a. Cuerpo con forma de rombo, mucho más alto en la porción media del cuerpo (a nivel del origen de la aleta dorsal), ligeramente comprimido lateralmente; dientes ausentes (sin dientes); ojo mediano, de diámetro mayor que la longitud de la boca; serie longitudinal con entre 36 y 37 escamas ... Curimatidae (representada en las aguas continentales e insulares de Costa Rica por una única especie, el "Capani, Capaniz, Carpa o Plateada" - Cyphocharax magdalenae, distribuida en la región Pacifico Sur; Fig. $1 \mathrm{~N}$ )

18b. Cuerpo alargado, de altura más o menos uniforme a lo largo del cuerpo, robusto, no comprimido lateralmente; ojo relativamente pequeño, de diámetro generalmente menor que la longitud de la boca; dientes presentes (con dientes); serie longitudinal con entre 26 y 27 escamas ... Lebiasinidae (representada en las aguas continentales e insulares de Costa Rica por una única especie, la "Candela o Candelilla" - Lebiasina boruca, distribuida en la región Pacifico Sur; Fig. 2B)

19a. Aletas pélvicas ausentes o si presentes fusionadas o modificadas en forma de una ventosa ventral (Figs. 9B-D) ... 20

19b. Aletas pélvicas presentes e individualizadas, no fusionadas o modificadas en forma de una ventosa ventral (e.g., Fig. 5C) ... 22

20a. Cuerpo robusto y ovalado a redondeado en vista lateral, elástico o flácido en apariencia y con la capacidad de expandirse o inflarse; boca con dos placas dentarias en cada mandíbula formando una especie de "pico"; aletas pélvicas ausentes; una única aleta dorsal ... Tetraodontidae (representada en las aguas continentales e insulares de Costa Rica por tres especies, conocidas 
como "Peces globo, Tamboriles, Timboriles, Timburiles o Botetes", presentes en ambientes costeros en el Atlántico y Pacífico; Fig. 4J)

20b. Cuerpo generalmente alargado en vista lateral, no elástico, flácido, expandible ni inflable; dientes individualizados, no fusionados en placas dentarias, pequeños y generalmente cónicos (Fig. $8 \mathrm{~A})$, distribuidos en hileras en ambas mandíbulas; aletas pélvicas presentes, fusionadas o modificadas en forma de una ventosa (Figs. 9B-D); una o dos aletas dorsales ... 21

21a. Cuerpo desnudo (sin escamas); cuerpo con forma de gota en vista dorsal, el ancho de la cabeza (en vista dorsal) es generalmente mucho mayor que el ancho de la porción posterior del cuerpo; aletas pectorales unidas al disco adhesivo, formando la parte superior del disco (Fig. 9D); una aleta dorsal ... Gobiesocidae (representada en las aguas continentales e insulares de Costa Rica por 3 especies, conocidas como "Chupapiedras o Guarasapos", distribuidas en todo el país; Fig. 2L)

21b. Cuerpo cubierto por escamas, usualmente pequeñas; cuerpo generalmente cilíndrico o tubular, en vista dorsal el ancho de la cabeza es similar o ligeramente menor al de la porción posterior del cuerpo; aletas pectorales libres o separadas, no unidas al disco adhesivo (Figs. 9B-C); dos aletas dorsales ... Gobiidae (representada en las aguas continentales e insulares de Costa Rica por 22 especies, conocidas como "Chupapiedras o Gobios", distribuidas en todo el país; Fig. 2N)

22a. Dos aletas dorsales claramente separadas (e.g., Figs. 1D, 2M, 3B-D, 3I) o "una" sola aleta dorsal discontinua con una porción espinosa y otra mayoritariamente radiada claramente separadas (e.g., Fig. 3J) ... 23

22b. Una sola aleta dorsal, generalmente continua (e.g., Figs. 1A, 2D-E, 2G-H) o con una comisura leve o moderada (e.g., Figs. $1 \mathrm{~K}, 3 \mathrm{E}, 3 \mathrm{~F}, 3 \mathrm{~K}$ ) a relativamente fuerte (e.g., Fig. 1J) separando las porciones espinosa y radiada ... 29

23a. Línea lateral ausente; escamas generalmente lisas, cicloideas (Fig. 6C); aletas pélvicas situadas completamente por delante del origen de la primera aleta dorsal en el plano vertical (e.g., Figs. 1D, $2 \mathrm{M}, 3 \mathrm{~B}) \ldots 24$

23b. Línea lateral presente (ver Figs. 5A y 5D), conspicua; escamas generalmente ásperas, ctenoideas (Fig. 6D); aletas pélvicas situadas por debajo o detrás del origen de la primera aleta dorsal en el plano vertical (e.g., Figs. 3I-J), excepto en algunas especies de Carangidae (Fig. 3C) y Centropomidae (Fig. 3D) ... 26

24a. Cuerpo marrón, generalmente con pintas o manchas rojas, negras, verdes, doradas y/o moradas; distancia entre ambas aletas dorsales menor que la longitud de la base de la primera aleta dorsal; origen de la aleta pélvica ubicado por delante de la línea media de la aleta pectoral (extendida sobre el cuerpo y vista en el plano vertical); aleta caudal redondeada o convexa (Fig. 7C) a ligeramente truncada (Fig. 7D) ... Eleotridae (representada en las aguas continentales e insulares de Costa Rica por 15 especies, conocidas como "Guavinas, Pocoyos, Dormilones, Peces perro", distribuidas en todo el país; Fig. 2M)

24b. Cuerpo plateado o amarillento, generalmente sin manchas; distancia entre ambas aletas dorsales mayor que la longitud de la base de la primera aleta dorsal; origen de la aleta pélvica ubicado por debajo o por detrás (posterior) de la línea media de la aleta pectoral (extendida sobre el cuerpo y vista en el plano vertical); aleta caudal cóncava o ligeramente bifurcada (Figs. 7E-F) ... 25 

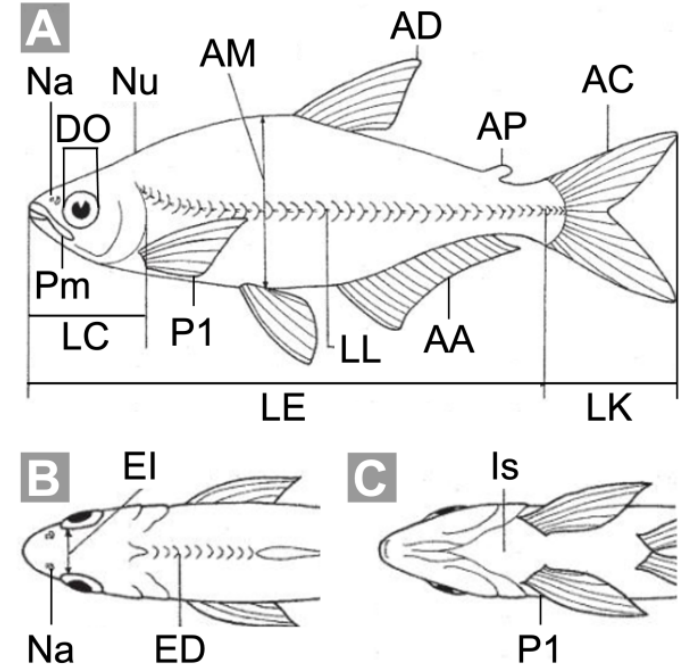

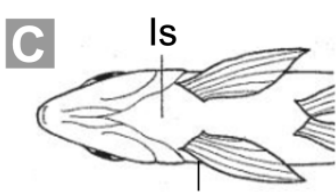

P1
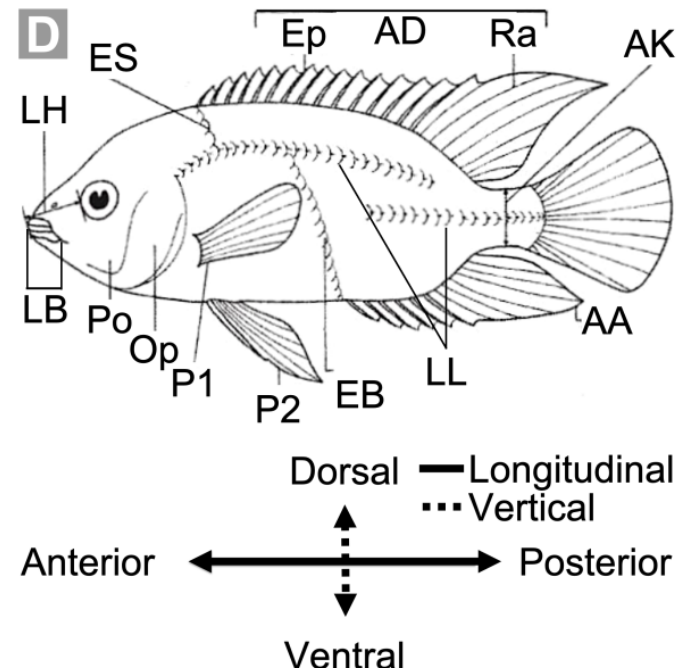

Fig. 5. Morfología externa generalizada de $(A-C)$ un miembro de la familia Characidae (Astyanax) y (D) un miembro de la

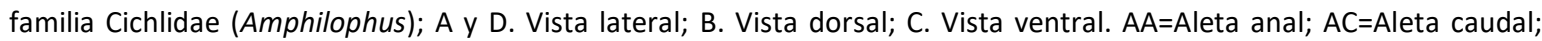
AK=Altura o profundidad máxima del pedúnculo caudal (medida en vista lateral y en línea recta desde el borde ventral al borde dorsal del pedúnculo caudal, que incluye la porción posterior del cuerpo entre en final de la aleta anal y el origen

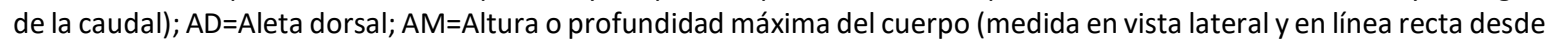
el borde ventral al borde dorsal de cuerpo en la porción más alta o profunda del pez); AP=Aleta dorsal adiposa (aleta suave y carnosa, desprovista de elementos óseos, situada posterior a la aleta dorsal y anterior a la aleta caudal; presente solo en algunos grupos de peces óseos); DO=Diámetro ocular, del ojo o de la órbita (medida desde el extremo anterior hasta el extremo posterior del ojo); EB=Escamas bajo la línea lateral (conteo de escamas que incluye las hileras o filas transversales, desde de la línea lateral, sin contar esta, hasta la poción ventral del cuerpo); ED=Escama predorsales (conteo de escamas, en el borde dorsal del cuerpo, entre el borde posterior de la cabeza y el origen de la aleta dorsal); El=Espacio interorbital (medida, en vista dorsal, entre los bordes dorsales de ambos ojos); Ep=Espinas (elementos óseos usualmente rígidos, no segmentados ni ramificados); ES=Escamas sobre la línea lateral (conteo de escamas que incluye las hileras o filas transversales, desde la línea lateral, sin contar esta, hasta la poción dorsal del cuerpo); Is=Istmo; LB=Longitud de la boca (medida desde el extremo anterior hasta el extremo posterior de la misma); LC=Longitud cefálica o de la cabeza (medida desde el extremo anterior de la boca hasta el borde posterior del opérculo); LE=Longitud estándar (medida desde el extremo anterior de la boca hasta el origen o base de la aleta caudal); LH=Longitud del hocico (medida desde el extremo anterior de la boca hasta el borde anterior del ojo); LK=Longitud de la cola (medida desde el origen o base de la aleta caudal hasta el extremo posterior de la misma); LL=Línea lateral [estructura a modo de canal o línea, continua/completa (A) o interrumpida (B), conspicua o tenue, presente en el costado de los peces, desde el borde posterior de la cabeza hasta la base de la cola o incluyendo esta]; $L T=L o n g i t u d$ total ( $L E+L K$, medida desde el extremo anterior de la boca hasta el extremo posterior de la aleta caudal); $\mathrm{Na}=\mathrm{Narina}$; $\mathrm{Nu}=\mathrm{Nuca}$ (borde antero-dorsal de la cabeza); Op=Opérculo; $\mathrm{P} 1=\mathrm{Aleta}$ pectoral; P2=Aleta pélvica; Pm=Premaxilar; Po=Preopérculo; Ra=Radios (elementos óseos usualmente flexibles, segmentados y ramificados). Modificado de Bussing (1998). 

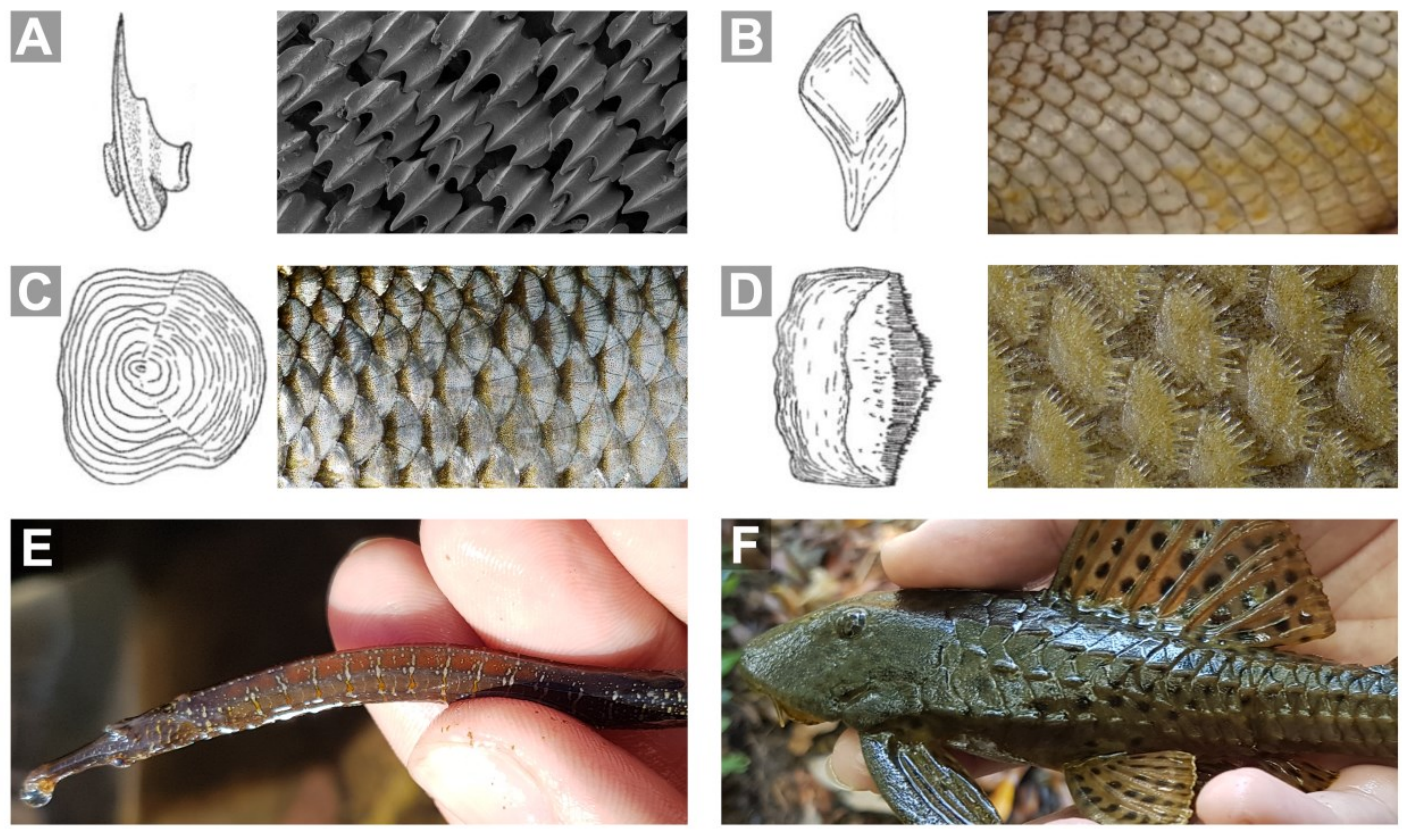

Fig. 6. Tipos de escamas en peces; A. Placoideas (presentes en tiburones y rayas); B. Ganoideas (presentes en el "Gaspar o Pejelagarto" - Atractosteus tropicus); C. Cicloideas (de textura lisa); D. Ctenoideas (en forma de peine, de textura áspera); E. Escamas modificadas como anillos, formando una coraza rígida (e.g.,"Pez pipa" - Pseudophallus elcapitanensis); F. Escamas modificadas como placas, formando una coraza rígida (e.g., "Pez diablo o Pleco" - Hypostomus aspidolepis). Modificado de Bussing (1998); E y F: Fotografías inéditas.
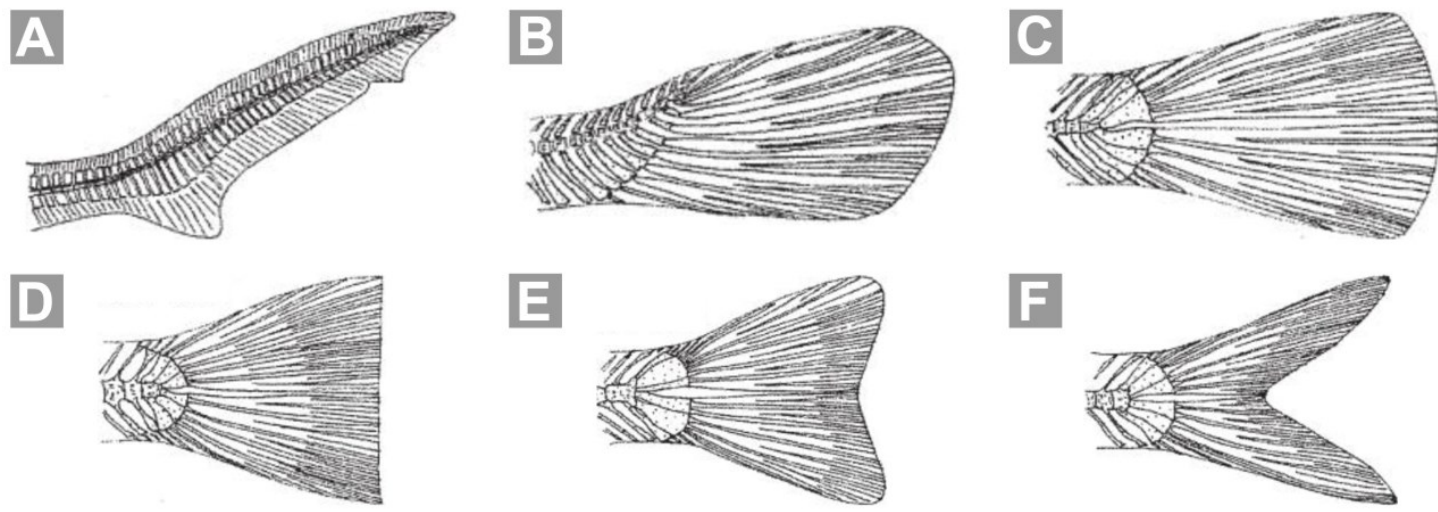

Fig. 7. Tipos de aleta caudal en peces; A. Heterocerca (en tiburones y rayas); B. Heterocerca abreviada (en el "Gaspar o Pejelagarto" - Atractosteus tropicus); C. Homocerca redondeada o convexa; D. Homocerca truncada; E. Homocerca recortada o cóncava; F. Homocerca bifurcada, bilobulada o ahorquillada. Modificado de Bussing (1998).

25a. Cuerpo alargado y ligeramente comprimido lateralmente, con ejemplares adultos no sobrepasando los $20 \mathrm{~cm}$ de longitud total; cuerpo con una franja lateral plateada, gruesa, generalmente delineada en su porción dorsal por una franja negra más delgada; origen de la primera aleta dorsal situado en o por detrás de la línea media del cuerpo en el plano vertical, generalmente por detrás del origen de la aleta anal; aleta caudal bifurcada (Fig. 7F) ... Atherinopsidae (representada en las aguas continentales e insulares de Costa Rica por 16 especies, conocidas como "Sardinas, Sardinas plateadas, Plateadas, Pejerreyes o Alfileres", distribuidas en todo el país; Fig. 1D) 
25b. Cuerpo ligeramente alargado, robusto, no comprimido lateralmente, con ejemplares adultos fácilmente sobrepasando los $20 \mathrm{~cm}$ de longitud total; sin una franja lateral plateada delineada en su porción dorsal por una franja negra más delgada; origen de la primera aleta dorsal situado por delante de la línea media del cuerpo en el plano vertical, siempre por delante del origen de la aleta anal; aleta caudal recortada, cóncava o ligeramente bifurcada (Figs. 7E-F)... Mugilidae (representada en las aguas continentales e insulares de Costa Rica por seis especies, conocidas como "Lisas, Machines, Tepemechines, Sabaletes o Bobos", distribuidas en todo el país; Fig. 3B)

26a. Mandíbula superior proyectada anteriormente (sobrepasa claramente la mandíbula inferior en el plano vertical; Fig. 8I); boca grande, con su extremo posterior claramente sobrepasando el límite posterior del ojo en el eje vertical; aleta pectoral dividida en dos porciones, con la porción ventral (ubicada por debajo de las branquias) presentando varios (usualmente siete) filamentos libres; distancia entre ambas aletas dorsales igual o mayor que la longitud de la base de la primera aleta dorsal ... Polynemidae (representada en las aguas continentales e insulares de Costa Rica por dos especies, conocidas como "Ratones, Bobos o Barbudos barbú" - Polydactylus approximans y $P$. virginicus, presentes en ambientes costeros en el Pacífico y Atlántico, respectivamente; Fig. 3I)

26b. Mandíbula superior no proyectada anteriormente (excepto en algunas especies de Sciaenidae, donde se presenta ligeramente proyectada anteriormente o de igual tamaño que la mandíbula inferior); boca mediana a grande, con su extremo posterior generalmente no sobrepasando el límite posterior del ojo en el eje vertical (excepto en algunas especies de Carangidae; Fig. 3C); aleta pectoral no dividida en dos porciones y sin filamentos libres en la región ventral; distancia entre ambas aletas dorsales (o ambas porciones de la aleta dorsal, i.e., espinosa y radiada) menor que la longitud de la base de la primera aleta dorsal ... 27

27a. Escamas pequeñas, reducidas, no visibles a simple vista (excepto las de la porción posterior de la línea lateral que están modificadas en forma de placas espinosas bastante evidentes); pedúnculo caudal estrecho, su altura máxima equivale a menos de un $20 \%$ de la altura máxima del cuerpo (ver Figs. 5A, 5D); aleta caudal fuertemente ahorquillada (Fig. 7F); línea lateral no extendiéndose en la aleta caudal (ver Fig. 5D) ... Carangidae (representada en las aguas continentales e insulares de Costa Rica por nueve especies, conocidas como "Jureles, Palometas, Pámpanos o Cojinoas", presentes en ambientes costeros en el Atlántico y Pacífico; Fig. 3C)

27b. Escamas grandes, no reducidas, visibles a simple vista, no modificadas en forma de placas en la porción posterior de la línea lateral; pedúnculo caudal no necesariamente estrecho, su altura máxima equivale a más de un $20 \%$ de la altura máxima del cuerpo (ver Figs. 5A, 5D); aleta caudal variable (cóncava, truncada, recortada, convexa o bifurcada; Figs. 7C-F), no fuertemente ahorquillada; línea lateral extendiéndose total o parcialmente en la aleta caudal (ver Figs. 3D, 3J) ... 28

28a. Cabeza terminada en punta y con la frente cóncava; mandíbula inferior fuertemente proyectada anteriormente (sobrepasa claramente la mandíbula superior en el plano vertical; Fig. 8J); aleta anal con 3 espinas fuertes (ver Figs. 5D y 9A); aleta caudal bifurcada (Fig. 7F) ... Centropomidae (representada en las aguas continentales e insulares de Costa Rica por 11 especies, conocidas como "Róbalos, Robalos o Gualajes", presentes en ambientes costeros en el Atlántico y Pacífico; Fig. 3D)

28b. Cabeza no necesariamente terminada en punta, con la frente convexa o ligeramente cóncava; mandíbula inferior no fuertemente proyectada anteriormente (esta condición es variable en miembros de esta familia, con algunas especies con ambas mandíbulas equidistantes, o alguna de las dos ligeramente proyectada anteriormente respecto a la otra); aleta anal con 1-2 espinas fuertes 
(ver Figs. 5D y 9A); aleta caudal de forma variable, nunca fuertemente bifurcada ... Sciaenidae (representada en las aguas continentales e insulares de Costa Rica por cinco especies, conocidas como "Corvinas o Verrugate(o)s", presentes en ambientes costeros en el Atlántico y Pacífico; Fig. 3Ј)

29a. Escamas ausentes; cirros dérmicos (prolongaciones o apéndices relativamente pequeños) presentes sobre el ojo y en las narinas anteriores ... Blenniidae (representada en las aguas continentales e insulares de Costa Rica por una única especie, el "Cachudito o tambollo de aleta manchada" - Hypsoblennius maculipinna, presente en ambientes costeros en el Pacífico; Fig. 1G)

29b. Escamas presentes; sin cirros dérmicos en la cabeza ... $\mathbf{3 0}$

30a. Cuerpo generalmente alargado, su altura máxima generalmente representa menos de un 30\% de la longitud estándar del cuerpo (medida desde la punta del hocico hasta el origen de la aleta caudal, sin contar esta), excepto en algunas especies de Clupeidae; aleta dorsal relativamente corta, la longitud de su base representa mucho menos de la mitad de la longitud estándar del cuerpo (medida desde la punta del hocico hasta el origen de la aleta caudal, sin contar esta), y generalmente restringida a la mitad posterior del cuerpo (su origen se sitúa por detrás de la línea media del cuerpo en el eje vertical), excepto en algunas especies de Albulidae (Fig. 1A), Clupeidae (Fig. 2C) y Engraulidae (Fig. 2E) ... 31

30b. Cuerpo alto, no necesariamente alargado, su altura máxima generalmente representa más de un $30 \%$ de la longitud estándar del cuerpo (medida desde la punta del hocico hasta el origen de la aleta caudal, sin contar esta), excepto en algunas especies de Dactyloscopidae (Fig. 1H) y Microdesmidae (Fig. 20); aleta dorsal relativamente larga, la longitud de su base representa más de la mitad (o casi la mitad) de la longitud estandar del cuerpo (medida desde la punta del hocico hasta el origen de la aleta caudal, sin contar esta), con su origen generalmente en o por delante de la línea media del cuerpo en el eje vertical ... 43

31a. Cuerpo desnudo (sin escamas); barbillas o barbillones presentes ... Trichomycteridae (representada en las aguas continentales e insulares de Costa Rica por una única especie, la "Laucha, Babosa, Barbudo o Pez gato lápiz" - Trichomycterus striatus, distribuida en la región Pacífico Sur; Fig. 4G)

31b. Cuerpo cubierto por escamas, ganoideas (Fig. 6B), cicloideas (Fig. 6C) o ctenoideas (Fig. 6D); barbillas o barbillones ausentes ... 32

32a. Origen de la aleta dorsal situado en la porción posterior del cuerpo, generalmente cerca de la aleta caudal, y por detrás del origen de la aleta anal en el eje vertical (por delante del origen de la aleta anal en las especies de Hemiramphidae; Fig. 1F) ... 33

32b. Origen de la aleta dorsal situado cerca de la línea media del cuerpo en el eje vertical, por delante del origen de la aleta anal (por detrás del origen de la aleta anal en algunas especies de Poeciliidae; Fig. 2H) ... 37 
A
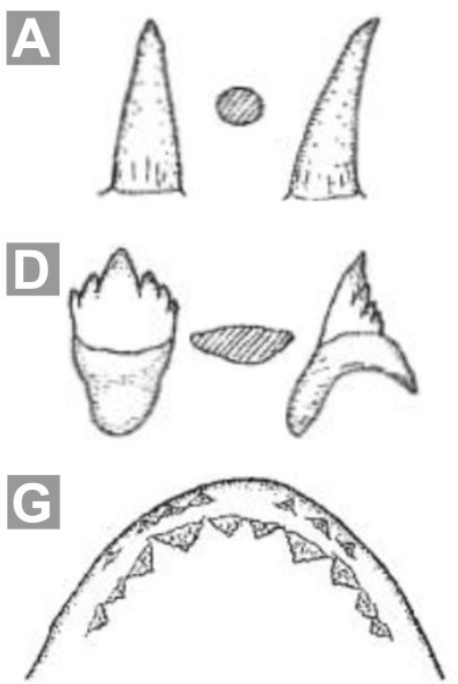
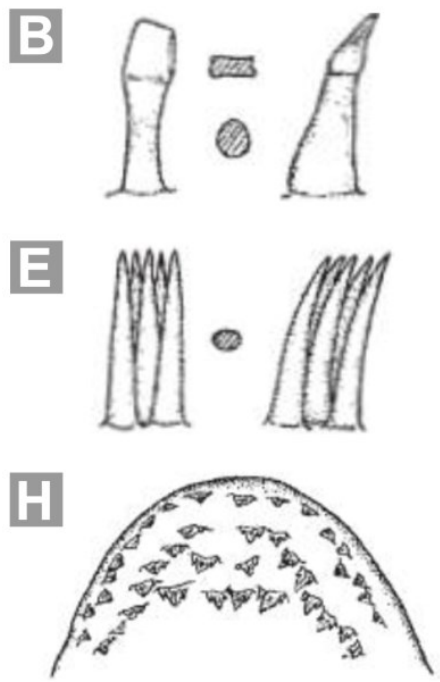
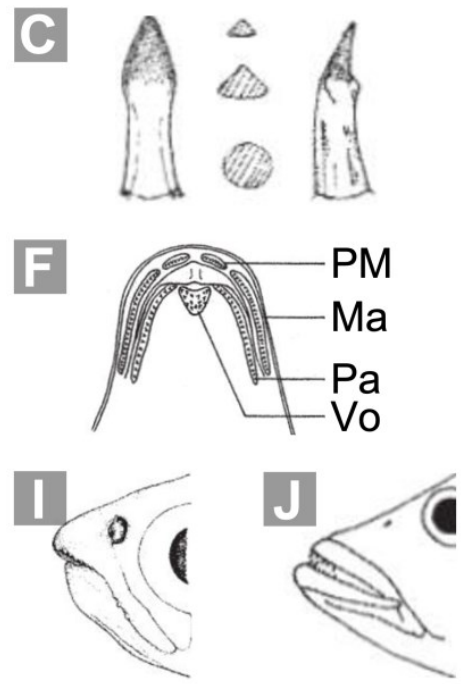

Fig. 8. Tipos de dentición en peces y disposición mandibular. En A-E, de izquierda a derecha, vistas frontal, transversal (corte) y lateral; A. Cónico; B. Truncado; C. Espatulado; D. Multicúspide; E. Filiforme. F. Vista ventral de la mandíbula superior de un pez óseo generalizado mostrando diferentes conjuntos dentales: PM=Premaxilar; Ma=Maxilar; $\mathrm{Pa}=$ Palatino; $\mathrm{Vo}=$ Vomerino. $\mathrm{G}$. Vista ventral de la mandíbula superior de un miembro de la familia Characidae, mostrando dos hileras o filas de dientes multicúspides; $\mathrm{H}$. Vista ventral de la mandíbula superior de un miembro de la familia Bryconidae (Brycon), mostrando cuatro hileras o filas de dientes multicúspides. I. Vista lateral de un miembro de la familia Bryconidae (Brycon) mostrando prognatismo superior, i.e., la mandíbula superior proyectada anteriormente; J. Vista lateral de un miembro de la familia Cichlidae (Parachromis) mostrando prognatismo inferior, i.e., la mandíbula inferior proyectada anteriormente. Modificado de Bussing (1998).

33a. Una o ambas mandíbulas muy alargadas [su longitud igual o mayor que la longitud post-ocular de la cabeza (medida entre el límite posterior del ojo y el borde posterior del opérculo)], en forma de pico, y generalmente provistas con varios dientes, conspicuos, del tipo caninos (Fig. 8A) o en forma de aguja (Fig. 8E); aleta caudal heterocerca abreviada (Fig. 7B), recortada (Fig. 7E) o cóncava a bifurcada (Fig. 7F); adultos sobrepasando los $30 \mathrm{~cm}$ de longitud total, alcanzando incluso longitudes de más de $1 \mathrm{~m}$... 34

33b. Mandíbulas no muy alargadas [su longitud generalmente menor que la longitud post-ocular de la cabeza (medida entre el límite posterior del ojo y el borde posterior del opérculo)], con dientes pequeños, generalmente poco evidentes; aleta caudal redondeada (Fig. 7C); adultos generalmente no sobrepasando los $20 \mathrm{~cm}$ de longitud total, en muy pocos casos sobrepasando los $30 \mathrm{~cm}$ pero nunca los $35 \mathrm{~cm}$... 36

34a. Escamas ganoideas (Fig. 6B), bastante evidentes, formando una especie de coraza rígida que recubre la mayor parte del cuerpo; aletas pélvicas situadas en o por delante de la línea media del cuerpo en el eje vertical; aleta caudal heterocerca abreviada, con el borde distal redondeado o convexo (Fig. 7B); típicamente dulceacuícolas ... Lepisosteidae (representada en las aguas continentales e insulares de Costa Rica por una única especie, el "Gaspar o Pejelagarto" Atractosteus tropicus, distribuida en la región Norte y Caribe Norte; Fig. 3A)

34b. Escamas cicloideas (Fig. 6C), reducidas, no formando una coraza rígida; aletas pélvicas situadas por detrás de la línea media del cuerpo en el eje vertical; aleta caudal homocerca, recortada o cóncava a bifurcada (Figs. 6E-F); típicamente asociados a ambientes costero-estuarinos ... 35 

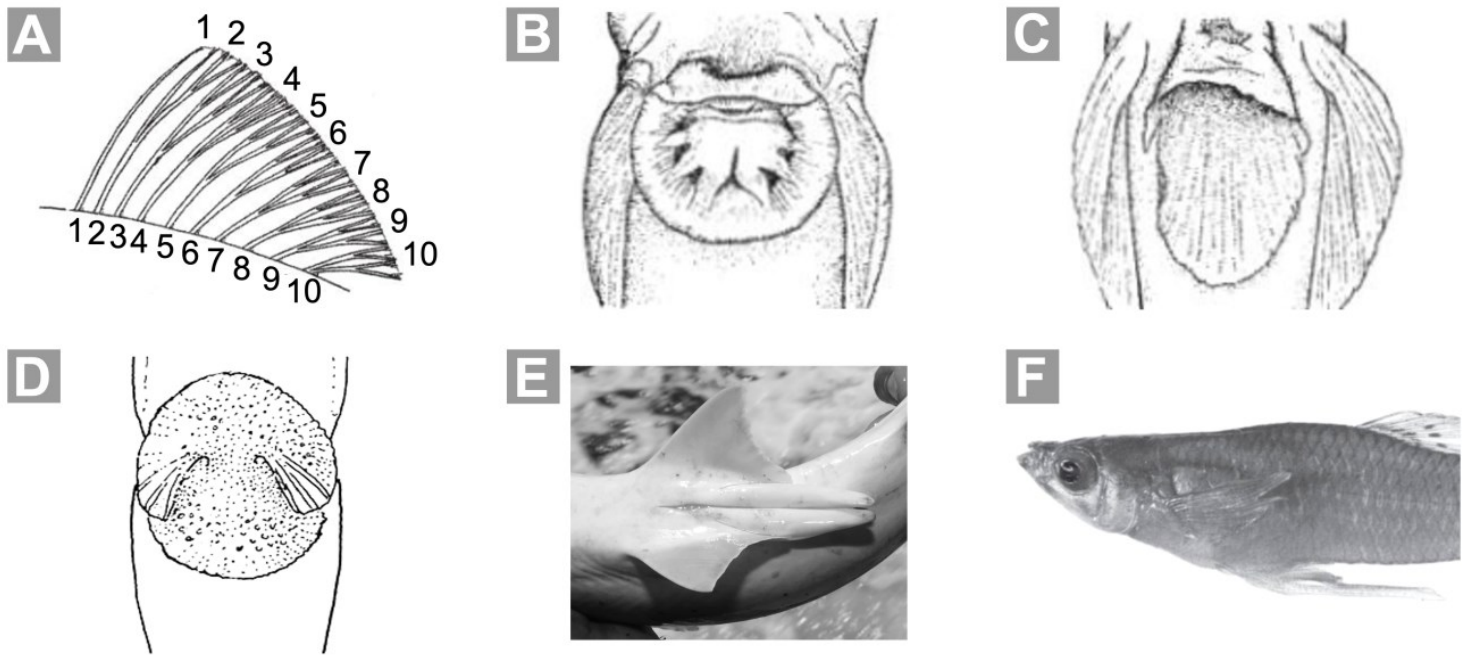

Fig. 9. Conteo de elementos (radios y espinas) asociados a las aletas [dorsal en el caso del ejemplo ilustrado; A (vista lateral)] y modificaciones en aletas pélvicas [B-D. Ventosa ventral en Gobiidae, Sicydium (B), Gobiidae, Awaous (C), y Gobiesocidae, Gobiesox (D); E. Pterigopodios en Carcharhinidae, Carcharhinus; vistas ventrales] y anal (F. Gonopodio en Poeciliidae, Gambusia; vista lateral). Modificado de Bussing (1998); E y F: Fotografías inéditas.

35a. Ambas mandíbulas alargadas; origen de la aleta dorsal situado por detrás del origen de la aleta anal en el eje vertical; aleta caudal truncada a recortada o ligeramente bifurcada (Figs. 7D-E) ... Belonidae (representada en las aguas continentales e insulares de Costa Rica por dos especies, conocidas como "Agujones, Agujas o Picudas" - Strongylura marina y S. timuco, presentes en ambientes costeros en el Atlántico; Fig. 1E)

35b. Mandíbula superior corta y de forma triangular en vista dorsal, mandibular inferior alargada; origen de la aleta dorsal situado en línea o por delante del origen de la aleta anal en el eje vertical; aleta caudal cóncava a bifurcada (Fig. 7F) ... Hemiramphidae (representada en las aguas continentales e insulares de Costa Rica por dos especies, conocidas como "Saltadores, Agujetas o Medio picos" - Hemiramphus brasiliensis e Hyporhamphus roberti, presentes en ambientes costeros en el Atlántico; Fig. 1F)

36a. Hocico relativamente alargado, su longitud corresponde al doble o casi el doble del diámetro del ojo; borde superior del ojo de color blanco o plateado, resaltando sobre el perfil dorsal de la cabeza; perfil predorsal recto (i.e., nuca plana o recta); aleta pélvica no reducida, su longitud (largo) similar a la longitud (largo) de la aleta pectoral ... Anablepidae (representada en las aguas continentales e insulares de Costa Rica por una única especie, el "Ojos blancos u Olomina de estero" - Oxyzygonectes dovii, presente en ambientes costeros en el Pacífico; Fig. 2G)

36b. Hocico relativamente corto, su longitud igual o similar al diámetro del ojo; borde superior del ojo de color variable, usualmente café, nunca blanco o plateado, situado dentro de los límites de la cabeza y no resaltando sobre el perfil dorsal; perfil predorsal ligeramente curvo, convexo (i.e., nuca ligeramente curva, no plana); aleta pélvica reducida, su longitud (largo) menor (40-75\%) que la longitud (largo) de la aleta pectoral ... Rivulidae (representada en las aguas continentales e insulares de Costa Rica por siete especies, conocidas como "Killis, Bobitas u Olominas saltonas", distribuidas en todo el país; Fig. 2l) 
37a. Cuerpo de coloración general variable, generalmente amarillenta o marrón, generalmente con manchas o marcas de colores (amarillo, rojo, café, naranja, verde-azulado, entre otros) en el cuerpo y/o aletas; aleta caudal

ligeramente convexa o redondeada (Fig. 7C); adultos generalmente no sobrepasando los $25 \mathrm{~cm}$ de longitud total, nunca sobrepasando los $40 \mathrm{~cm}$; típicamente dulceacuícolas ... 38

37b. Coloración general plateada, con un patrón de contra-sombreado (dorso relativamente oscuro y vientre claro), generalmente sin manchas o marcas de colores (fuera del negro, café y/o plateado) en el cuerpo y aletas; aleta caudal cóncava o bifurcada (Figs. 7E-F); adultos generalmente sobrepasando los $25 \mathrm{~cm}$ de longitud total [excepto en algunas especies de Clupeidae (Fig. 2D) y Engraulidae (Fig. 2E)], alcanzando incluso longitudes de más de $1 \mathrm{~m}$; típicamente asociados a ambientes costero-estuarinos ... 39

38a. Boca grande, su longitud mayor que el diámetro del ojo, con varios dientes caninos, largos y puntiagudos (Fig. 8A); aleta anal situada en posición posterior (bien por detrás de la línea media del cuerpo en el eje horizontal); machos con la aleta anal no modificada en forma de un órgano reproductivo copulatorio; adultos sobrepasando los $25 \mathrm{~cm}$ de longitud total, alcanzando incluso longitudes de hasta 35-40 cm ... Erythrinidae (representada en las aguas continentales e insulares de Costa Rica por una única especie, el "Pez perro, Pez tigre o Dientón" - Hoplias microlepis, distribuida en la región Pacífico Sur; Fig. 2A)

38b. Boca generalmente pequeña, su longitud menor que el diámetro del ojo (excepto en el "Gasparcillo, Pepesca gaspar, Olomina picuda, Picuda, Alumina picuda, Pepesca o Agujilla" Belonesox belizanus, Poeciliidae), con varios dientes diminutos, generalmente puntiagudos, filiformes (Fig. 8E); aleta anal situada generalmente situada en posición anterior (por delante o cerca de la línea media del cuerpo en el eje horizontal); machos con la aleta anal modificada en forma de un órgano reproductivo copulatorio (gonopodio; Fig. 9F); adultos generalmente no sobrepasando los $10 \mathrm{~cm}$ de longitud total, algunas pocas especies alcanzando hasta $15 \mathrm{~cm}$... Poeciliidae (representada en las aguas continentales e insulares de Costa Rica por 26 especies, conocidas como "Olominas, Aluminas, Bobitas, Chimbolas, Pepescas, Panzonas, Killis o Bubuchas", distribuidas en todo el país; Fig. $2 \mathrm{H}$ )

39a. Boca pequeña, su borde posterior generalmente no alcanza el borde anterior del ojo; borde ventral del cuerpo delgado, "afilado", presentando una quilla de escudetes (serie de escamas espinosas y duras) sensible al tacto ... Clupeidae (representada en las aguas continentales e insulares de Costa Rica por cuatro especies, conocidas como "Sardinas, Arenques, Machuelos, Piquitingas o Sardinetas", distribuidas la región Norte y en zonas costeras en el Atlántico y Pacífico; Fig. 2D)

39b. Boca grande, su borde posterior alcanza o sobrepasa el borde anterior del ojo (excepto en Albulidae; Fig. 1A); borde ventral del cuerpo no necesariamente delgado, no afilado, sin una quilla de escamas ... 40

40a. Boca relativamente pequeña, su borde posterior generalmente no alcanza el borde anterior del ojo; origen de las aletas pélvicas por detrás del origen de la aleta dorsal en el plano vertical; aleta anal relativamente pequeña, su base (longitud) menor (menos de 50\%) que la base de la aleta dorsal ... Albulidae (representada en las aguas continentales e insulares de Costa Rica por una única especie, el "Macabí" - Albula vulpes, presente en ambientes costeros en el Atlántico; Fig. 1A)

40b. Boca grande, su borde posterior alcanza o sobrepasa el borde posterior del ojo; origen de las aletas pélvicas por delante del origen de la aleta dorsal en el plano vertical; aleta anal relativamente grande, similar (más de un 50\%) o mayor que la longitud de la base de la aleta dorsal ... 41 
41a. Boca inferior, no terminal, hocico redondeado o cónico, con la mandíbula superior proyectada anteriormente (sobrepasa la mandíbula inferior en el plano vertical; Fig. 8I); sin una placa ósea alargada entre las mandíbulas inferiores; con una franja plateada (muy evidente) en los costados; adultos no sobrepasando los $35 \mathrm{~cm}$ de longitud total ... Engraulidae (representada en las aguas continentales e insulares de Costa Rica por cinco especies, conocidas como "Anchoas, Anchovetas o Boquerones", presentes en ambientes costeros en el Atlántico y Pacífico; Fig. 2E)

41b. Boca terminal, ligeramente en posición inferior, hocico no redondeado ni cónico, con ambas mandíbulas de aproximadamente el mismo tamaño o la inferior proyectada anteriormente (sobrepasa la mandíbula superior en el plano vertical Fig. 8J); con una placa ósea alargada entre las mandíbulas inferiores; generalmente sin una franja plateada en los costados (al menos no tan evidente); adultos sobrepasando los $40 \mathrm{~cm}$ de longitud total, alcanzando incluso longitudes de más de $1 \mathrm{~m}$... 42

42a. Hocico relativamente corto, su longitud menor que el diámetro del ojo (ver Figs. 5A-D); mandíbulas superior e inferior de aproximadamente el mismo largo; hueso premaxilar (ver Fig. 5A) relativamente delgado, su altura generalmente menor que el diámetro del ojo; escamas pequeñas, menores que el tamaño de la pupila; último radio de la aleta dorsal no prolongado en forma de un filamento ... Elopidae (representada en las aguas continentales e insulares de Costa Rica por una única especie, el "Machete o Pez torpedo" - Elops affinis, presente en ambientes costeros en el Pacífico; Fig. 2J)

42b. Hocico relativamente largo, su longitud igual o mayor que el diámetro del ojo (ver Figs. 5A-D); mandíbula inferior proyectada anteriormente (sobrepasa la mandíbula superior en el plano vertical Fig. 8J); hueso premaxilar (ver Fig. 5A) prominente, su altura generalmente mayor que el diámetro del ojo; escamas grandes, generalmente mayores que el tamaño de la pupila; último radio de la aleta dorsal prolongado en forma de un filamento ... Megalopidae [representada en las aguas continentales e insulares de Costa Rica por una única especie, el "Sábalo, Sábalo real o Tarpón" Megalops atlanticus, originalmente distribuida en la vertiente Atlántica, no obstante con registros recientemente confirmados en zonas costeras en el Pacífico (Castellanos-Galindo et al. 2019); Fig. $2 \mathrm{~K}]$

43a. Cuerpo alargado, su altura máxima (ver Fig. 5A) generalmente representa menos de un $30 \%$ de la longitud estándar (medida desde la punta del hocico hasta el origen de la aleta caudal, sin contar esta); boca fuertemente oblicua, con la mandíbula inferior proyectada anteriormente (sobrepasa la mandíbula superior en el plano vertical Fig. 8J); aletas pélvicas reducidas compuestas por una sola espina y tres radios blandos (ver Figs. 5D y $9 \mathrm{~A}$ ), mucho menores que las aletas pectorales y con su origen o inserción anterior a estas en el plano vertical, generalmente por debajo de la cabeza (i.e., anterior al límite posterior del preopérculo en el plano vertical); aleta dorsal larga, compuesta por 35 o más elementos (ver Fig. 9A) ... 44

43b. Cuerpo alto, no necesariamente alargado, su altura máxima (ver Fig. 5A) generalmente representa más de un $30 \%$ de la longitud estándar (medida desde la punta del hocico hasta el origen de la aleta caudal, sin contar esta); boca no necesariamente oblicua, con ambas mandíbulas del mismo tamaño o con la superior proyectada anteriormente (sobrepasa la mandíbula inferior en el plano vertical; Fig. 81); aletas pélvicas no reducidas, de tamaño similar o ligeramente menor que las aletas pectorales y con su origen o inserción por debajo o posterior a estas en el plano vertical, generalmente atrás de la cabeza (i.e., posterior al límite posterior del preopérculo en el plano vertical); aleta dorsal relativamente corta, generalmente compuesta por menos de 35 elementos (ver Fig. 9A) ... 45 
44a. Cabeza grande y más ancha que el resto del cuerpo, dorso-ventralmente aplanada; ojos salientes y pedunculados, situados por encima de la cabeza; aleta dorsal conformada por 35-41 elementos (ver Fig. 9A); aletas dorsal y anal no confluentes (i.e., no unidas o conectadas) con la aleta caudal ... Dactyloscopidae (representada en las aguas continentales e insulares de Costa Rica por una única especie, el "Miraestrellas estuarino o Miraestrellas ribereño" - Dactyloscopus amnis, presente en ambientes costeros en el Pacífico; Fig. $1 \mathrm{H}$ )

44b. Cabeza proporcional al resto del cuerpo, no dorso-ventralmente aplanada; ojos adheridos a la cabeza, no pedunculados, situados a los lados de la cabeza; aleta dorsal conformada por 53-60 elementos (ver Fig. 9A); aletas dorsal y anal confluentes (i.e., unidas o conectadas) con la aleta caudal ... Microdesmidae (representada en las aguas continentales e insulares de Costa Rica por una única especie, el "Gobio-gusano de dorso manchado o Pez lombriz de lomo punteado" Microdesmus dorsipunctatus, presente en ambientes costeros en el Pacífico; Fig. 20).

45a. Mandíbula inferior proyectada anteriormente (sobrepasa la mandíbula superior en el plano vertical; Fig. 8J); extremo posterior de la boca claramente sobrepasando el límite posterior del ojo en el plano vertical; región opercular (ver Fig. 5D) sin espinas rígidas; aleta dorsal en apariencia discontinua, con una profunda comisura

separando las porciones espinosa y radiada ... Centrarchidae (representada en las aguas continentales e insulares de Costa Rica por una única especie, la "Lobina negra o Perca americana" - Micropterus salmoides, distribuida en el valle Central, región Norte y Caribe Norte, en cuerpos de agua dulce artificiales; esta es una especie introducida y poco frecuente en ambientes naturales; otras especies de la familia podrían encontrarse también, como introducidas, en cuerpos de agua artificiales o lagunas privadas; Fig. 1J).

45b. Mandíbula inferior generalmente no proyectada anteriormente [ligeramente proyectada anteriormente en algunos miembros de Cichlidae (Fig. 8J) y Serranidae (Fig. 3K)]; extremo posterior de la boca generalmente no sobrepasando el límite posterior del ojo en el plano vertical [excepto en Serranidae (Fig. 3K), una familia asociada a ambientes costero-estuarinos]; región opercular (ver Fig. 5D) con (e.g., Fig. 3K) o sin (e..g., Fig. 5D) espinas rígidas; aleta dorsal sin una comisura fuerte separando las porciones espinosa y radiada [excepto en algunas especies de Gerreidae (Fig. 3E) y Haemulidae (Fig. 3F)] ... 46

46a. Línea lateral incompleta, interrumpida (Fig. 5D); aleta dorsal generalmente con 15 o más espinas (ver Figs. 5D y 9A); aleta anal con 4 o más espinas (ver Figs. 5D y 9A), excepto en la "Mojarrita o Chogorra" - Andinoacara coeruleopunctatus y otras especies no nativas (e.g., Oreochromis "Tilapia") que presentan 3 espinas anales ... Cichlidae (representada en las aguas continentales e insulares de Costa Rica por 26 especies, conocidas como "Mojarras, Chogorras, Viejas, Mogas, Tilapias, Congos o Guapotes", distribuidas en todo el país; restringidas a aguas dulces, la "Vieja, Mojarra, Pis pis, Mojarra de estero, Palometa, Mojarra azul o Tilapia" - Vieja maculicauda se encuentra también en aguas salobres en zonas costeras en el Atlántico; Fig. 2C).

46b. Línea lateral completa, ininterrumpida (e.g. Fig. 5A); aleta dorsal con menos de 15 espinas (ver Figs. 5D y 9A); aleta anal con 2-3 espinas (ver Figs. 5D y 9A) ... 47 (especies típicamente asociadas a ambientes costero-estuarinos, excepto miembros de Cyprinidae).

47a. Boca relativamente pequeña, con su extremo posterior no alcanzando el límite anterior del ojo en el plano vertical; región opercular (ver Fig. 5D) lisa, no aserrada, sin espinas fuertes ... $\mathbf{4 8}$ (especies típicamente marinas o dulceacuícolas estrictas). 
47b. Boca pequeña a relativamente grande, con su extremo posterior alcanzando o sobrepasando el límite anterior del ojo en el plano vertical; región opercular (ver Fig. 5D) aserrada y/o con una o más espinas prominentes ... $\mathbf{5 0}$ (especies típicamente asociadas a ambientes costero-estuarinos).

48a. Barbillas presentes (Cyprinus carpio - "Carpa común"; Fig. 2F) o ausentes (Carassius auratus "Carpa dorada"); dentición faríngea (i.e., en la región postero-dorsal de la cavidad bucal) presente; aleta dorsal con 3-4 espinas (ver Figs. 5D y 9A) no muy fuertes, relativamente flexibles; coloración general del cuerpo dorado, naranja-rojizo o cobrizo-plateado ... Cyprinidae (representada en las aguas continentales e insulares de Costa Rica por dos especies introducidas, conocidas como "Carpas", restrictas a ambientes dulceacuícolas y presentes en cuerpos de agua artificiales y ambientes naturales alterados en el valle Central y región Norte; Fig. 2F)

48b. Barbillas ausentes; dentición faríngea (i.e., en la región postero-dorsal de la cavidad bucal) presente o ausente; aleta dorsal con 8-14 espinas (ver Figs. 5D y 9A) generalmente fuertes y/o rígidas; coloración general variable, raramente dorado o naranja-rojizo ... 49 (especies típicamente asociadas a ambientes costero-estuarinos).

49a. Longitud de la cabeza menor que la altura máxima del cuerpo (entre un 60 y $80 \%$ de esta); ojo de diámetro similar o mayor que la longitud de la boca; dientes incisivos a truncados (Fig. 8B); dentición faríngea (i.e., en la región postero-dorsal de la cavidad bucal) ausente; cuerpo ovalado, más alto en su porción media; aleta caudal cóncava a ahorquillada (Figs. 7E-F) ... Kyphosidae (representada en las aguas continentales e insulares de Costa Rica por una única especie, la "Chopa blanca" - Kyphosus sectatrix, presente en ambientes costeros en el Atlántico; Fig. 1K).

49b. Longitud de la cabeza similar o ligeramente mayor que la altura máxima del cuerpo; en adultos, ojo de diámetro similar o menor que la longitud de la boca; dientes caninos (Fig. 8A); dentición faríngea (i.e., en la región postero-dorsal de la cavidad bucal) presente, bien desarrollada, en forma de placas; cuerpo alargado, de altura similar en la mayor parte del tronco; aleta caudal ligeramente recortada a convexa o redondeada (Figs. 7C-D) ... Labridae (representada en las aguas continentales e insulares de Costa Rica por tres especies, conocidas como "Señoritas", presentes en ambientes costeros en el Atlántico y Pacífico; Fig. 3G).

50a. Aleta dorsal con 9-10 espinas (ver Figs. 5D y 9A); porción espinosa de la aleta dorsal (su base) similar o menos larga que la (base de la) porción radiada (ligeramente más larga en algunos miembros de Lutjanidae) ... 51

50b. Aleta dorsal con 11-14 espinas (ver Figs. 5D y 9A); porción espinosa de la aleta dorsal (su base) mucho más larga que la (base de la) porción radiada ... 52

51a. Hocico relativamente corto, su longitud (cuando la boca esta cerrada, no proyectada anteriormente) generalmente menor que el diámetro del ojo (ver Figs. 5A y 5D); dientes diminutos, filiformes (Fig. 8E); dentición palatina (paladar) ausente (ver Fig. 8F); aleta caudal fuertemente ahorquillada (Fig. 7F); cuerpo generalmente plateado, uniforme, sin manchas o barras conspicuas (excepto en las especies de Eugerres y Gerres las cuales presentan barras o franjas negras), aletas transparentes, gris oscuro y/o amarillentas con o sin los extremos negros (principalmente la dorsal) ... Gerreidae (representada en las aguas continentales e insulares de Costa Rica por 14 especies, conocidas como "Mojarras, Comearenas, Petaca banderitas o Palmito(a)s", presentes en ambientes costeros en el Atlántico y Pacífico; Fig. 3E).

51b. Hocico relativamente largo, su longitud (cuando la boca esta cerrada, no proyectada anteriormente) igual o mayor que el diámetro del ojo (ver Figs. 5A y 5D); dientes medianos, cónicos, caninos (Fig. 8A); dentición palatina bien desarrollada, en forma de parche (ver Fig. 8F); aleta caudal 
generalmente truncada a recortada (Figs. 7D-E), no fuertemente ahorquillada; coloración general variable (raramente plateado uniforme), con tonos amarillentos, rojizos, cobrizos, grisáceos y/o marrones, con o sin manchas o barras de tonos variables (negro, marrón, azul, entre otros) ... Lutjanidae (representada en las aguas continentales e insulares de Costa Rica por ocho especies, conocidas como "Pargos", presentes en ambientes costeros en el Atlántico y Pacífico; Fig. 3H).

52a. Dientes pequeños, cónicos, no caninos; opérculo con una espina prominente; aleta dorsal con 12-14 espinas (ver Figs. 5D y 9A); aleta caudal cóncava a ahorquillada (Figs, 7E-F); coloración general variable, sin manchas rojo-marrón en todo el cuerpo ... Haemulidae (representada en las aguas continentales e insulares de Costa Rica por ocho especies, conocidas como "Roncadores", presentes en ambientes costeros en el Atlántico y Pacífico; las dos especies del género Rhonciscus penetran considerables distancias en el cauce de los ríos; Fig. 3F)

52b. Dientes medianos o grandes, bastante evidentes, caninos (Fig. 8A); opérculo con 3 espinas prominentes; aleta con 11 espinas (ver Figs. 5D y 9A); aleta caudal truncada a ligeramente redondeada (Figs. 7C-D); coloración general variable entre rojomarrón/cobrizo/blanquecino/rosado o blanco-verdoso, con múltiples manchas rojo-marrón ... Serranidae (representada en las aguas continentales e insulares de Costa Rica por una única especie, la "Cabrilla colorada o Mero colorado" - Epinephelus guttatus, presente en ambientes costeros en el Atlántico; Fig. 3K).

\section{DISCUSIÓN}

En la última revisión disponible, incluyendo claves taxonómicas para la identificación de las principales familias de peces de las aguas continentales de Costa Rica, Bussing (1998) incluyó un total de 34 familias; revisiones recientes (e.g., Angulo et al., 2013; 2015; 2017; 2018; 2020, en prep.) indican, no obstante, que este número alcanza las 53 familias. Este incremento en el número total de familias listadas para el país responde a la adición de múltiples especies [y consecuentemente de la(s) familia(s) correspondiente(s)] así como a diversos avances en la taxonomía de ciertos grupos supra-específicos provocando alteraciones importantes en su estructura y composición [(e.g., la separación de Bryconidae de Characidae, a como considerado originalmente por Bussing (1998), ver Abe et al., (2014)]. En la presente revisión, tomando como base las publicaciones supracitadas se incluyeron y consideraron la totalidad de familias actualmente reconocidas y listadas para el país (i.e., 53), lo cual significó un incremento de más de un $56 \%$ en el número total de familias tratadas en claves previas, esto al menos tomando como base la publicación de Bussing (1998). Publicaciones anteriores al trabajo de Bussing, e.g., López-Sánchez (1968), incluyeron un total de 31 familias, tomando esto como base, la presente contribución significa un incremento de más de un $71 \%$ en el número total de familias tratadas.

La presente contribución no constituye un tratamiento completo sobre la fauna íctica del país, a nivel de grupos supra-específicos, ya que obvia o excluye mucha información en detalle; por esta razón no reemplaza trabajos previos, como el de Bussing (1998), entre otros, los cuales ofrecen información (aún) válida y detallada sobre aspectos ecológicos (comportamentales y de alimentación), otros caracteres útiles para identificación, patrones de distribución latitudinal y altitudinal, entre otros, relacionados con este componente de la diversidad costarricense. Dado esto, la presente contribución constituye más un complemento y/o actualización a tales obras. Otras publicaciones, incluyendo las bases de datos en línea de Robertson \& Allen (2015) y Robertson \& Van Tassell (2019), así como la de Froese \& Pauly (2020), ofrecen también información de gran relevancia, actualizada y de utilidad a efectos de identificación; las dos primeras, centradas particularmente en el componente marino y periférico (i.e., familias típicamente marinas con 
miembros que ocasionalmente ingresan, o pasan parte importante de su ciclo de vida, a/en ambientes dulceacuícolas), Pacífico y Atlántico respectivamente, y la tercera un tanto más general, incluyendo a la mayoría de los grupos tratados en la presente contribución. Por otro lado, trabajos relativamente recientes ofrecen además claves enfocadas en áreas específicas del país, por lo cual se recomienda su uso a escala local; en ese sentido, cabe resaltar las publicaciones de Angulo et al. (2015) para la cuenca del río Tusubres (Pacífico Central), Angulo et al. (2017) para la región de Sarapiquí, Heredia (Caribe norte) y Angulo et al. (2020) para la cuenca del río Coto (Pacífico sur), entre otros.

\section{AGRADECIMIENTOS}

Los autores agradecen a la dirección y personal técnico y administrativo de la Escuela de Biología, el Museo de Zoología, el Centro de Investigación en Biodiversidad y Ecología Tropical (CIBET) y el Centro en Investigación de Ciencias del Mar y Limnología (CIMAR) de la Universidad de Costa Rica (UCR) por los apoyos y facilidades (espacio, equipos, materiales, entre otros) brindados. Esta contribución es parte del proyecto 808-C1-125-Peces de la aguas continentales e insulares de Costa Rica: diversidad, distribución, sistemática, taxonomía y estado actual de conservación, con AA como investigador principal, inscrito y financiado en parte por la Vicerrectoría de Investigación de la UCR por medio del CIMAR. Carlos A. Garita-Alvarado y dos colegas anónimos realizaron una revisión crítica del manuscrito y brindaron sugerencias importantes para su mejora. Terry C. Grande y Mark V. H. Wilson facilitaron el acceso y concedieron el permiso de uso para las ilustraciones presentadas en las figuras 1-4.

\section{ÉTICA, CONFLICTO DE INTERESES Y DECLARACIÓN DE FINANCIAMIENTO}

Los autores declaran haber cumplido con todos los requisitos éticos y legales pertinentes, tanto durante el estudio como en el manuscrito; que no hay conflictos de interés de ningún tipo, y que todas las fuentes financieras se detallan plena y claramente en la sección de agradecimientos. Asimismo, están de acuerdo con la versión editada final del documento. El respectivo documento legal firmado se encuentra en los archivos de la revista.

La declaración de la contribución de cada autor al manuscrito es la siguiente: A.A.,: ideó el trabajo, recopiló los datos, redactó el manuscrito y editó y unió las figuras; A. R.R.C., \& M.L.,: apoyaron en la recopilación de datos e información literaria, revisaron el manuscrito y realizaron ediciones y sugerencias varias para su mejora. 


\section{REFERENCIAS}

Abe, K. T., Mariguela, T. C., Avelino, G. S., Foresti, F., \& Oliveira, C. (2014). Systematic and historical biogeography of the Bryconidae (Ostariophysi: Characiformes) suggesting a new rearrangement of its genera and an old origin of Mesoamerican ichthyofauna. BMC Evolutionary Biology, 14(152), 1-15. DOI: 10.1186/1471-2148-14-152

Álvarez-Calderón, F. S., Herrera, D., \& Angulo, A. (2018). First record of the highfin goby Gobionellus oceanicus (Pallas, 1770) (Gobiiformes: Gobiidae) in Costa Rican freshwaters. Cuadernos de Investigación UNED, 10(2), 404-408. DOI: 10.22458/urj.v10i2.2169

Angulo, A. (2013) Nombres comunes y técnicos de los peces de agua dulce de Costa Rica. Filología y Lingüística, 39(2), 77103. DOI: $10.15517 /$ RFL.V39I2.15061

Angulo, A., \& Gracian-Negrete, J. M. (2013). A new species of Brycon (Characiformes: Characidae) from Nicaragua and Costa Rica, with a key to the lower Mesoamerican species of the genus. Zootaxa, 3731(2), 255-266. DOI: 10.11646/zootaxa.3731.2.6

Angulo, A., Garita-Alvarado, C. A., Bussing, W. A., \& López, M. I. (2013). Annotated checklist of the freshwater fishes of continental and insular Costa Rica: additions and nomenclatural revisions. Check list, 9(5), 987-1019. DOI: $10.15560 / 9.5 .987$

Angulo, A., Molina-Arias, A., Murase, A., Miyazaki, Y., Bussing, W. A., \& López, M. I. (2015). Fishes from the Tusubres River basin, Pacific coast, Costa Rica: Checklist, identification key and photographic album. Check list, 11(3), 1666, 115. DOI: $10.15560 / 11.3 .1666$

Angulo, A., López, M. I., Bussing, W. A., Ramírez-Coghi, A. R., \& Arias-Godínez, G. (2016). Colección ictiológica del Museo de Zoología de la Universidad de Costa Rica. En Del Moral-Flores L. F., Ramírez-Villalobos, A. J., Martínez-Pérez, J. A., González-Acosta, A. F. \& Franco-López, J. (Eds.), Colecciones Ictiológicas de Latinoamérica (pp. 56-65). México: Editorial de la Facultad de Estudios Superiores Iztacala, Universidad Nacional Autónoma de México \& Sociedad Mexicana de Ictiología.

Angulo, A., Naranjo-Elizondo, B., Rojas, E., \& Ley-López, J. M. (2017). Fishes from the Tirimbina Biological Reserve, La Virgen de Sarapiquí, Heredia, Costa Rica. Check List, 13(5), 683-702. DOI: 10.15560/13.5.683

Angulo, A., Santos, A. C., López, M. I., Langeani, F., \& McMahan, C. D. (2018a). A new species of Astyanax Baird \& Girard, 1854 (Characiformes: Characidae) from Costa Rica and Panama, with a key to the lower Central American species of the genus. Journal of Fish Biology, 92(6), 1866-1887. DOI: 10.1111/jfb.13626

Angulo, A., Donascimiento, C., Lasso-Alcalá, O. M., Farah-Pérez, A., Langeani, F., \& McMahan, C. D. (2018b). Redescription of Trichomycterus striatus (Meek \& Hildebrand, 1913) (Siluriformes: Trichomycteridae), with notes on its geographical distribution. Zootaxa, 4420(4), 530-550. DOI: 10.11646/zootaxa.4420.4.5

Angulo, A., San Gil-León, J., Oviedo, A., Abarca-Odio, J. I., \& Umaña-Villalobos, G. (2020). The fishes from the Coto River basin, Pacific coast, Costa Rica. Zootaxa, 4751(1), 1-28. DOI: 10.11646/zootaxa.4751.1.1

Arguedas, J. C. V. (2019). Diversidad de peces de agua dulce en la zona costera de los distritos Bahía Ballena y Puerto Cortés del cantón de Osa, zona sur de Costa Rica. Posgrado y Sociedad. Revista Electrónica del Sistema de Estudios de Posgrado, 17(2), 17-32. DOI: 10.22458/rpys.v17i2.2201

Bussing, W. A. (1998). Peces de las aguas continentales de Costa Rica. Freshwater fishes of Costa Rica. San José: Editorial Universidad de Costa Rica.

Castellanos-Galindo, G. A., Robertson, D. R., Pacheco, B., Angulo, A., \& Chong, C. (2019). Atlantic Tarpon in the Tropical Eastern Pacific 80 years after it first crossed the Panama Canal. Reviews in Fish Biology and Fisheries, 29, 401416. DOI: 10.1007/s11160-019-09565-z

Fricke, R., Eschmeyer, W. N., \& Van der Laan, R. (2020). Eschmeyer's Catalog of Fishes: Genera, Species, References. Recuperado de http://researcharchive.calacademy.org/research/ichthyology/catalog/fishcatmain.asp. 
Froese, R., \& Pauly, D. (2020). FishBase. Recuperado de http://fishbase.org

López-Sánchez, M. I. (1968). Clave para los peces de las aguas continentales de Costa Rica. San José: Editorial de la Facultad de Ciencias y Letras de la Universidad de Costa Rica.

Matamoros, W. A., Chakrabarty, P., Angulo, A., Garita-Alvarado, C. A., \& McMahan, C. D. (2013). A new species of Roeboides (Teleostei: Characidae) from Costa Rica and Panama, with a key to the Middle American species of the genus. Neotropical Ichthyology, 11(2), 285-290. DOI: 10.1590/S1679-62252013000200006

Myers, G. S. (1949). Salt-tolerance of fresh-water fish groups in relation to zoogeographical problems. Bijdragen tot de Dierkunde, 28(1949), 315-322.

Nelson, J. S., Grande, T. C., \& Wilson, M. V. (2016). Fishes of the World. New Jersey: John Wiley \& Sons.

Ota, R. R., Carvalho, F. R., \& Pavanelli, C. S. (2020). Taxonomic review of the Hyphessobrycon panamensis species-group (Characiformes: Characidae). Zootaxa, 4751(3), 401-436. DOI: 10.11646/zootaxa.4751.3.1

Robertson, D. R., \& Allen, G. R. (2015). Shorefishes of the Tropical Eastern Pacific: online information system. Version 2.0 Smithsonian Tropical Research Institute, Balboa, Panamá. Recuperado de https://biogeodb.stri.si.edu/sftep/en/pages

Robertson, D. R., \& Van Tassell, J. (2019). Shorefishes of the Greater Caribbean: online information system. Version 2.0 Smithsonian Tropical Research Institute, Balboa, Panamá. Recuperado de https://biogeodb.stri.si.edu/caribbean/en/pages

Rodríguez-Arias, C., Echavarría, M. Z., \& Chaves, D. A. (2019). Primer registro de tres especies de peces en el Río Grande de San Ramón, Costa Rica. Cuadernos de Investigación UNED, 11(3), 309-312. DOI: 10.22458/urj.v11i3.2608

Schmitter-Soto, J. J. (2017). A revision of Astyanax (Characiformes: Characidae) in Central and North America, with the description of nine new species. Journal of Natural History, 51(23-24), 1331-1424. DOI: 10.1080/00222933.2017.1324050 